\title{
Utilisation industrielle actuelle et potentielle des plasmas : synthèses, traitement des poudres, traitements métallurgiques, traitements de surface
}

\author{
P. Fauchais \\ Laboratoire de Thermodynamique, U.E.R. des Sciences, Université de Limoges, France
}

(Reçu le 20 février 1980, révisé le 28 avril 1980, accepté le 6 mai 1980)

\begin{abstract}
Résumé. - Après un bref historique de l'évolution de l'utilisation des plasmas au cours des 20 dernières années, nous nous proposons de rappeler sommairement ce qu'est un plasma en précisant notamment la notion d'équilibre et de décrire rapidement les différents types de plasmas utilisés; arc électrique, haute fréquence, micro-ondes, décharges couronnes, décharges luminescentes, décharges ioniques en indiquant leurs caractéristiques principales, leurs coûts et les quantités de produits que l'on peut traiter. Nous présenterons alors les problèmes posés par les réactions chimiques en plasma en insistant sur le mélange des réactifs et sur la trempe, puis nous caractériserons les réactions en phase gazeuse et les réactions en phase hétérogène. Nous aborderons ensuite les traitements de surface en plasma : carburation et nitruration, traitement des surfaces polymériques, gravure par plasma, dépots de films organiques et inorganiques, traitements de surface par pulvérisation cathodique et ion plating. La dernière partie de cet exposé, de loin la plus importante, sera consacrée aux applications industrielles actuelles et potentielles de la chimie des plasmas. Nous commencerons par situer le problème énergétique dans la conjoncture actuelle avant de passer aux applications que nous classerons en fonction de la valeur ajoutée et du tonnage produit. Dans les applications déjà industrialisées, nous verrons successivement pour les produits à moyenne valeur ajoutée : la sphéroïdisation, la refusion (fours à sole et à lingots), la production des particules ultrafines (pigments en particulier), la projection de divers matériaux (oxydes, carbures, borures, siliciures, cermets, composites...) et pour les produits à forte valeur ajoutée : les circuits électroniques, les traitements de surfaces organiques et inorganiques, les applications dans le domaine de la métrologie. Enfin nous examinerons les procédés potentiellement industrialisables : tout d'abord les produits à moyenne valeur ajoutée : acétylène, oxydes d'azote, production des ferroalliages et du silicium solaire, valorisation des minerais, puis les produits à moyenne ou forte valeur ajoutée : projections, traitements de surface, amélioration de la qualité des fibres naturelles, fabrication des fibres optiques, synthèses sélectives.
\end{abstract}

\begin{abstract}
After a brief historical description of the evolution of the uses of plasma during the last 20 years, we propose to recall summarily what is a plasma stating particularly the equilibrium concept and to describe briefly the different types of plasma used : arcs, high frequency, microwaves, corona discharges, glow discharges, ionic discharges and to indicate their main characteristics, their cost and the mass flow rates of products that can be treated. We will present then the problems that occur for the chemical reactions under plasma conditions : mixing of the reactants, quenching... either for the homogenous or heterogenous reactions. The surface treatments in plasma will be next treated : carburizing and nitriding, reactions with polymers, plasma etching, deposition and growth of organic and inorganic films, surface treatment by cathodic pulverization and ion plating. The last part of this paper, the most important, will be devoted to the industrial applications either actual or potential of plasma chemistry. We will start with the energetic problem before reviewing the applications that we will classify in function of the added value and of the quantity produced. About the actual industrial uses we will see successively for the mean added value products : spheroidization, remelting (batch or ingot melting), production of ultradispersed powders, spraying of various materials (oxides, carbides, borides, silicides, cermets, composites...) and for the high added value products : electronic circuits, surface treatment of organic and of inorganic substances, the application for metrology. At last we will examine the processes that are potentially usable in industry : first the products with a low or a mean added value : acetylene, nitric oxide, ferroalloys production, solar grade silicon production, complex ores processing and then the products with a mean or a high added value : spraying, surface treatments, treatments on natural materials (modification of adhesive properties, shrink proofing of wool and mohair...), optical fibers manufacture, selective synthesis.
\end{abstract}


1. Introduction. - La première réaction de chimie des plasmas en laboratoire fut la syn thèse de l'acétylène en 1797 par Henry et Dalton, encore qu'ils aient ignoré qu'ils faisaient de la chimie des plasmas avec leur décharge capacitive dans le méthane. Le premier procédé industriel, à la fin du $\mathrm{XIX}^{\mathrm{e}}$ siècle, fut la synthèse de l'oxyde d'azote par Birkeland et Eyde [1], dans un arc alternatif alimenté à l'air. Malheureusement les travaux empiriques effectués entre 1880 et 1910 sur les réactions chimiques dans les décharges électriques furent rapidement oubliés car alors le plasma était totalement inconnu et il était impossible de corréler les produits obtenus aux propriétés des décharges.

Il y a moins de 20 ans que les études sur la chimie des plasmas se sont considérablement développées. En effet les progrès effectués dans la connaissance du plasma ont permis une meilleure compréhension du rôle des diverses espèces le composant conduisant ainsi à une chimie prenant en compte non seulement les espèces neutres et les ions mais aussi les électrons, les radicaux libres, les espèces excitées (notamment les métastables avec une longue durée de vie souvent responsables de réactions spécifiques) et aussi, si nécessaire, les photons.

Vers les années 1970, avec l'amélioration des techniques de mesure, d'importants progrès ont été faits dans la compréhension des phénomènes et de la dynamique des réactions chimiques en plasma. Les mesures montrent d'ailleurs que dans la majorité des cas, les plasmas utilisés en chimie des plasmas sont hors d'équilibre. Les travaux effectués en physique moléculaire et atomique ou dans la chimie de la haute atmosphère ont permis d'améliorer la compréhension et la modélisation des réacteurs hors d'équilibre notamment en définissant mieux le rôle des cinétiques d'excitation électroniques, vibrationnelles et rotationnelles et en mesurant les déséquilibres entre ces différentes formes d'excitation [2-5].

2. La classification des plasmas. - 2.1 LE DEGRÉ D'ÉQUILIBRE DES PLASMAS. - Par définition, le plasma est un milieu constitué de molécules et d'atomes, le plus souvent dans des états excités, d'ions et d'électrons, l'ensemble étant électriquement neutre.

Une des classifications possibles repose sur le degré d'équilibre thermodynamique : complet (CTE), local (LTE), partiel (PLTE) et enfin totalement en déséquilibre. Cette connaissance du degré d'équilibre est très importante pour expliquer, avec le maximum d'exactitude, les divers phénomènes cinétiques rencontrés dans les réactions en plasma. Les plasmas utilisés sont essentiellement produits par des décharges électriques où les électrons libres acquièrent leur énergie du champ électrique imposé et la perdent par collision avec les molécules, les atomes ou les ions. Sauf en présence de flux d'électrons rapides et anisotropes, les décharges électriques aux propriétés physiques très différentes sont caractérisées par la densité $\left(n_{\mathrm{e}}\right)$ et l'énergie $\left(k T_{\mathrm{e}}\right)$ des électrons [6]. Sans entrer dans le détail des décharges électriques que nous verrons brièvement au prochain paragraphe, on peut cependant les classer sommairement en fonction des applications possibles à l'aide de deux paramètres : la pression et l'intensité du courant de décharge.

En général, lorsque la pression diminue, le rapport $T_{\mathrm{e}} / T_{\mathrm{h}}$ de la température des électrons à celle des particules lourdes, augmente de l'unité à la pression atmosphérique jusqu'à 100 environ pour des pressions inférieures au torr. Il en va de même lorsque l'on considère l'intensité du courant de décharge : plus de $50 \mathrm{~A}$ correspondent à un rapport voisin de 1 (pratiquement l'équilibre) et quelques $\mathrm{mA}$ à des rapports de 50 et plus. Avec des valeurs élevées de $T_{\mathrm{e}} / T_{\mathrm{h}}$ l'énergie des électrons est grande et la température du gaz voisine de l'ambiante. Ces plasmas sont donc parfaitement adaptés pour la chimie de matériaux sensibles aux effets de la température (composés organiques par exemple), cependant la puissance électrique est alors faible et la masse traitée limitée (quelques $\mathrm{mg} / \mathrm{s}$ ). Au contraire les plasmas proches de l'équilibre ont une température élevée (quelques milliers de degrés $\mathrm{K}$ ) et ils sont bien adaptés aux réactions qui nécessitent des transferts enthalpiques importants (fusion, sphéroïdisation, projection, vaporisation) ou pour la synthèse de matériaux inorganiques tels que les céramiques, les petites molécules (NO, $\mathrm{C}_{2} \mathrm{H}_{2}, \mathrm{HCN}$...). La puissance électrique peut atteindre $5 \mathrm{MW}$ et la masse traitée une centaine de $\mathrm{g} / \mathrm{s}$. Cependant les gradients de température et de densité sont élevés le long du rayon du plasma et il est difficile d'obtenir un traitement identique pour tout le matériau injecté. De plus dans la majorité des cas lorsque l'on injecte des réactifs froids dans le plasma au-delà des électrodes, le mélange résultant est hors d'équilibre.

2.2 LES PRINCIPAUX TYPES DE GÉNÉRATEURS A PLASMA UTILISÉs. - Nous examinerons sommairement successivement les arcs $(\sim 1 \mathrm{~atm})$, les décharges radio-fréquence $\left(10^{-3}<p<1 \mathrm{~atm}\right)$, les décharges micro-ondes $\left(10^{-2}<p<1 \mathrm{~atm}\right)$, les décharges couronnes $(p \sim 1 \mathrm{~atm})$, les décharges luminescentes $\left(10^{-4}<p<10^{-2} \mathrm{~atm}\right)$ et les décharges ioniques $\left(10^{-6}<p<10^{-4} \mathrm{~atm}\right)$

2.2.1 Les générateurs à arc électrique. - a) Courant continu. - La première configuration fut, en 1957, celle de Gage [7] où l'arc éclate entre une cathode centrale et une tuyère qui lui est concentrique.

Le confinement de l'arc est ainsi assuré par le refroidissement énergique de l'anode [8] et par l'écoulement de gaz froid qui s'établit autour de la colonne d'arc (le gaz étant introduit longitudinalement ou tangentiellement à la cathode). Un générateur de ce type (Fig. 1 [9]), fonctionnant en courant continu, a une puissance inférieure à $100 \mathrm{~kW}$ avec une anode en cuivre (dont la durée de vie est de 3 à $400 \mathrm{~h}$ [10]) 


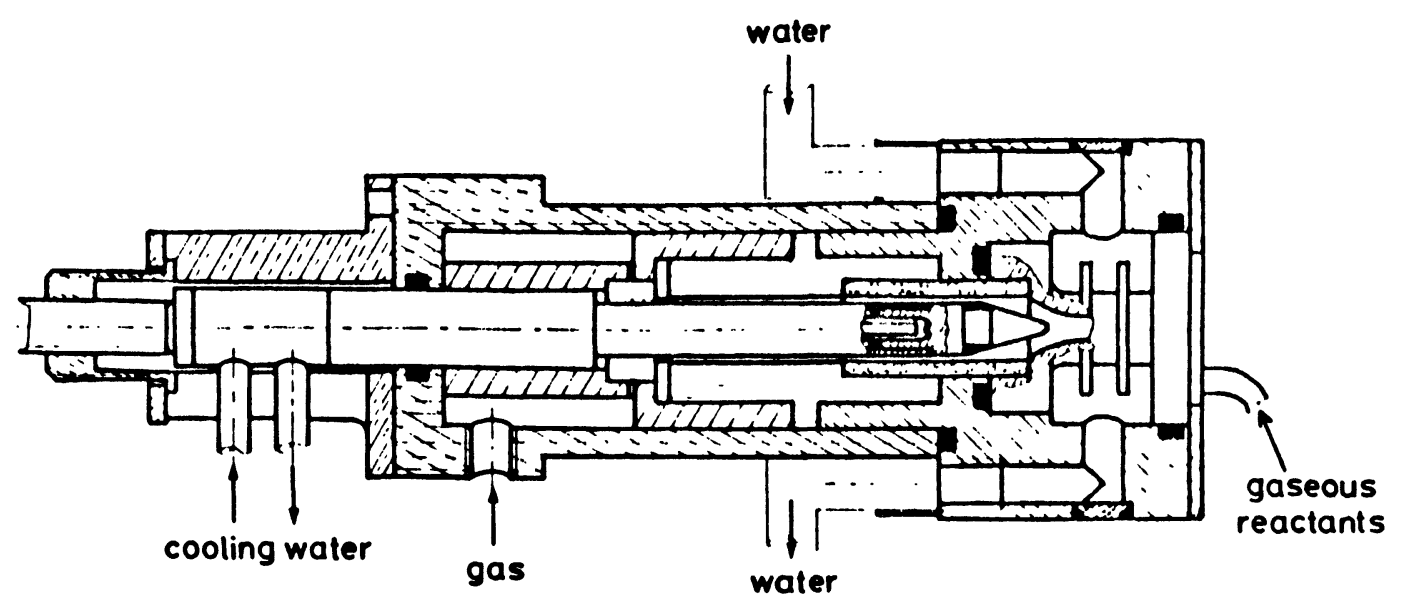

Fig. 1. - Générateur à plasma à courant continu .

[D.C. Plasma torch.]

et une cathode en tungstène (durée de vie de 150 à $200 \mathrm{~h}$ ) pour des gaz non oxydants ou en zirconium ou en hafnium pour des gaz oxydants (durée de vie de 30 à $50 \mathrm{~h}$ ). Ces générateurs utilisés en projection ou en découpage sont commercialisés par de nombreuses firmes : AVCO, METCO, ARCOS aux U.S.A., SNECMA en France, Swierk en Pologne ...

Pour des puissances supérieures, il convient d'abord d'améliorer le refroidissement des cathodes chaudes en les noyant par exemple dans une paroi de cuivre et d'utiliser soit des anodes à diamètres variables (Fig. 2a) dont la caractéristique $V, I$ a une pente croissante, soit des chambres d'arc à injection multiple (Fig. 2b) [11-13]. Enfin une stabilisation complémentaire est obtenue par la mise en rotation de l'arc dans la tuyère sous l'effet d'un champ magnétique. De tels générateurs peuvent atteindre $5 \mathrm{MW}$. Signalons enfin la possibilité d'utiliser des générateurs à eau ou à alcool comme celui de $250 \mathrm{~kW}$ de la société LONZA [14].

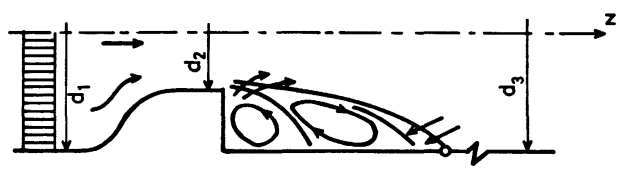

a)

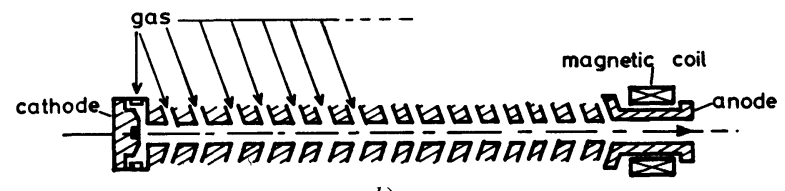

b)

Fig. 2. - Schémas de principe des générateurs à plasma de puissance $: a$ ) tuyères à changement de diamètre; $b$ ) générateur à arc constricté avec injections de gaz multiples.

[Principle of high power plasma generators : $a$ ) nozzle with change of diameter: $b$ ) constricted-arc gas heater with multi-injection.] b) Courant alternatif. - Les électrodes sont presque toujours en cuivre et la configuration est celle des électrodes tubulaires avec stabilisation tangentielle et magnétique, comme par exemple le générateur de Fey [15] de 1 MW (Fig. 3).

Afin d'éviter l'extinction de l'arc lors du changement de phase, une tension élevée à très haute fréquence est généralement superposée à l'arc [16, 17].

2.2.2 Les fours à arcs électriques. - L'inconvénient des générateurs décrits au paragraphe précédent est que, si les gaz sortant des tuyères ont une enthalpie élevée, une température qui peut dépasser $12000 \mathrm{~K}$ (Fig. 4 [2]), ces gaz ont également des vitesses qui varient entre 200 et $1500 \mathrm{~m} / \mathrm{s}$ (Fig. 5 [18]). De telles vitesses impliquent pour les matériaux solides introduits dans les jets de plasma des temps de séjour trop bref pour qu'ils y subissent autre chose qu'une simple fusion. La conception des fours va donc devoir d'une part améliorer le transfert de chaleur plasma particule et d'autre part surpasser la difficulté qu'il y a à introduire un corps solide dans un fluide très visqueux (environ 10 fois plus visqueux que le gaz froid [19]), sans oublier que dans les opérations industrielles il faut traiter en continu les produits avant et après réaction.

Beaucoup d'études bibliographiques [20-24] ont décrit de nombreux types de fours et leurs principales applications. Nous indiquerons ici simplement les quatre grands types de four tels qu'ils résultent de la classification de Bonet [25] reposant en partie sur le temps de séjour $t_{\mathrm{s}}$.

a) Fours à effet Maecker $\left(t_{\mathrm{s}} \sim 10^{-3} \mathrm{~s}\right)$. - Ces fours utilisent l'effet magnéto-hydrodynamique qui crée une zone de dépression en pointe de cathode [26-27] permettant un entraînement des particules dans le plasma. Dans les dispositifs d'Ion-arc [28], de Sheer [29], de Sayce [30] ou de Noranda [31], l'injection se fait autour de la cathode, l'arc se refermant sur 3 anodes de carbone consommables (Fig. 6), 


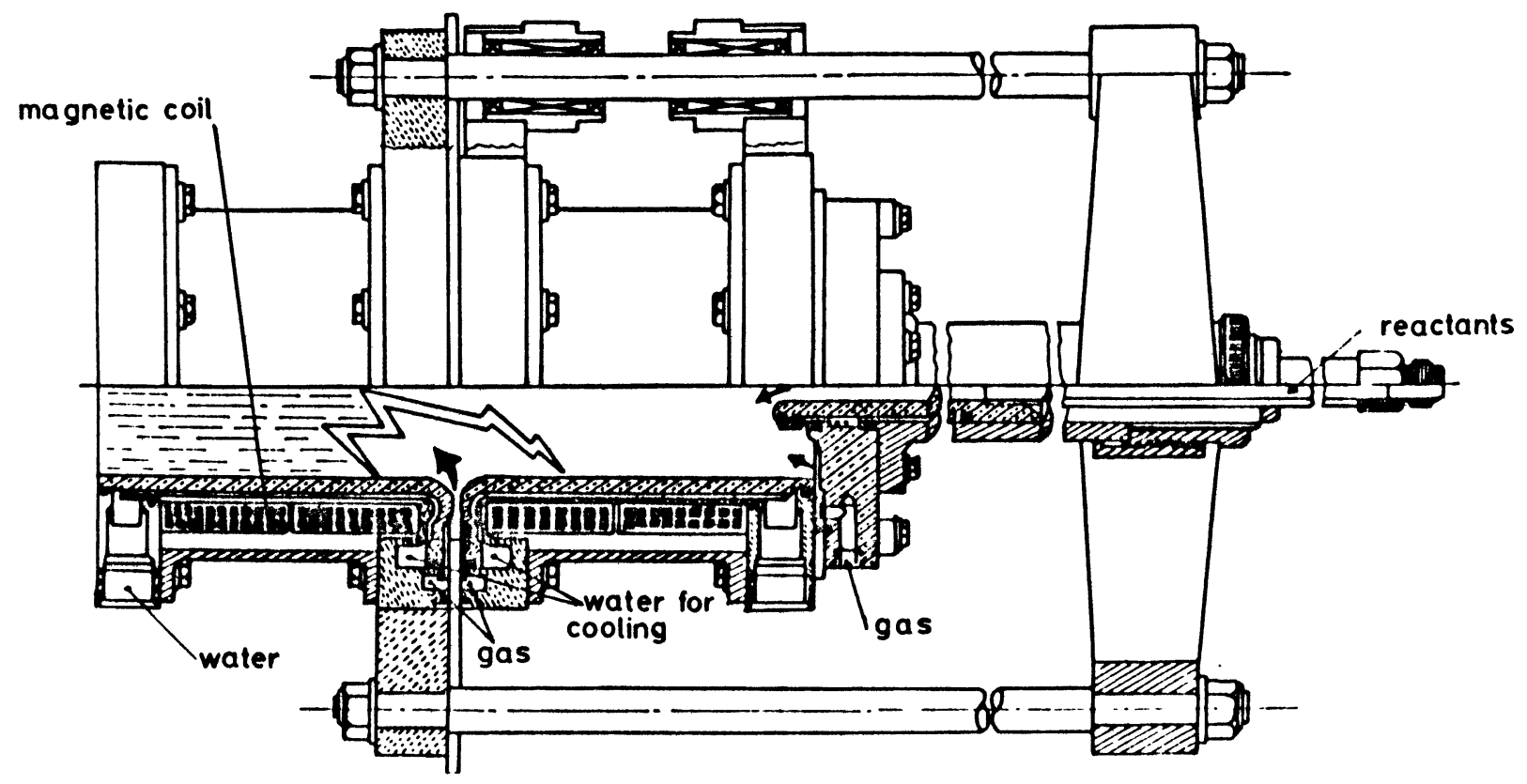

Fig. 3. - Réacteur à plasma de $1 \mathrm{MW}$ en courant alternatif monophasé (Westinghouse).

[A 3.5 MW single-phase alternating-current plasma torch (Westinghouse).]

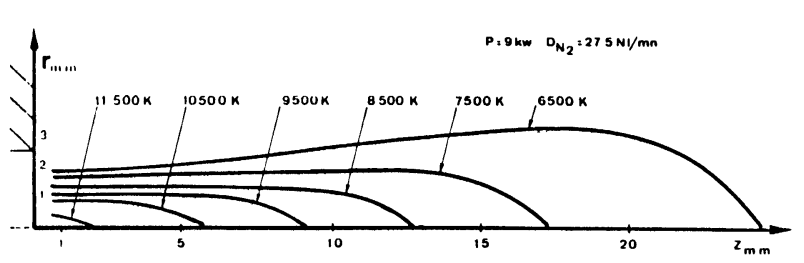

Fig. 4. - Isothermes d'un jet de plasma produit par un générateur alimenté en courant continu.

[Temperature contours in a D.C. nitrogen plasma jet.]

a)

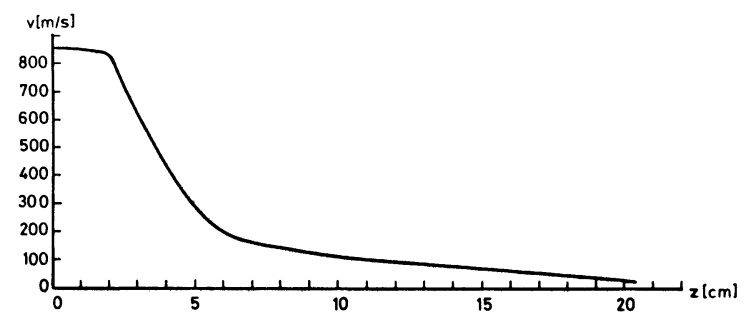

b)

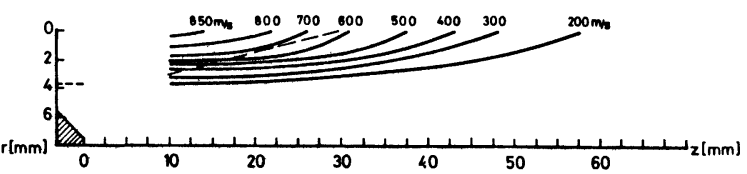

Fig. 5. - Distribution des vitesses d'écoulement dans un jet de plasma produit par un générateur alimenté en courant continu.

[Velocity distribution in a D.C. plasma-jet.]

sur 3 anodes poreuses, sur 3 jets de plasma, sur un bain liquide servant d'anode.

b) Fours à faible vitesse de passage des particules $\left(10^{-2}<t_{\mathrm{s}}<1 \mathrm{~s}\right)$. - On distingue les fours à sédimentation à co-courant [32] ou à contre-courant [33]

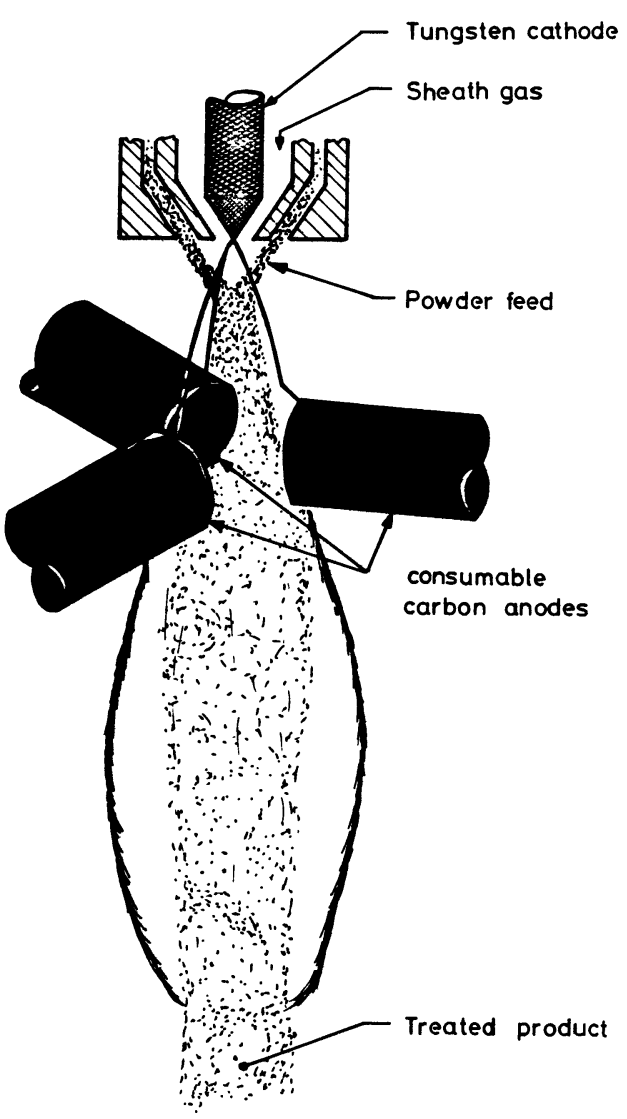

Fig. 6. - Four à plasma d'ion-arc pour la sphéroïdization des particules de zircone.

[Ion-arc heater for solid particles treatment (spheroidization of $\mathrm{ZrO}_{2}$ ).]

comme celui de Segworth à électrodes consommables, qui avec une puissance de $40 \mathrm{~kW}$, permet la réduction de la chromite (Fig. 7), les fours à expansion du 


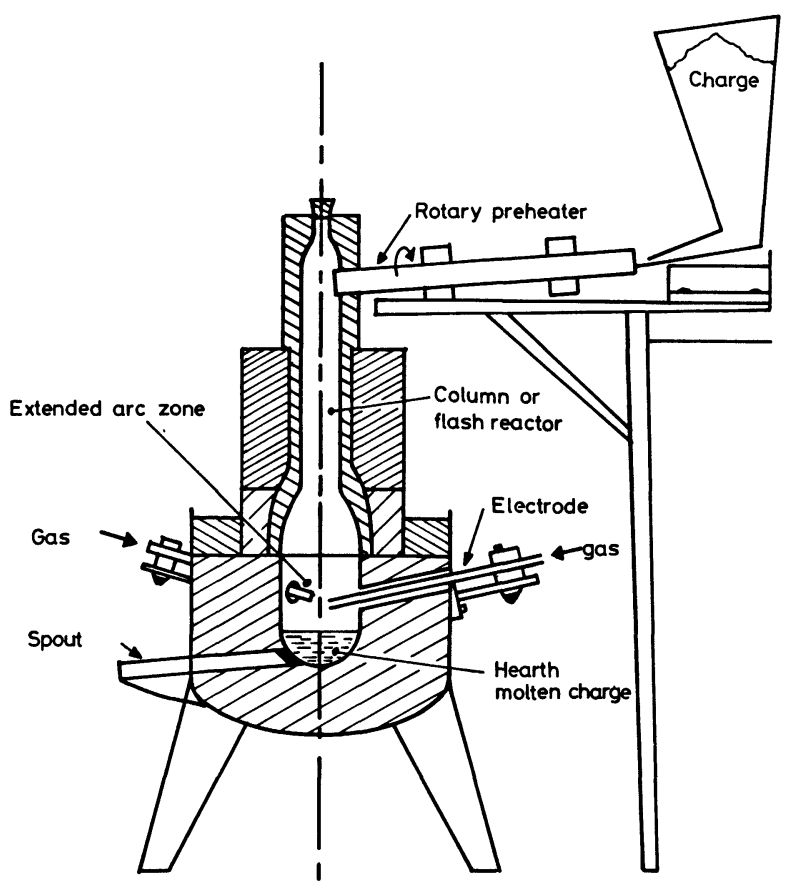

Fig. 7. - Réacteur plasma triphasé pour la réduction de la chromite.

[Extended arc flash reactor (EAFR) for chromite reduction.]

volume plasma par rotation de la paroi [34], de la cathode [35] ou par arc continu sous champ magnétique [36].

c) Fours à parois tournantes ou ruisselantes $\left(t_{\mathrm{s}}>1 \mathrm{~s}\right) .-$ Les fours à parois tournantes sont à

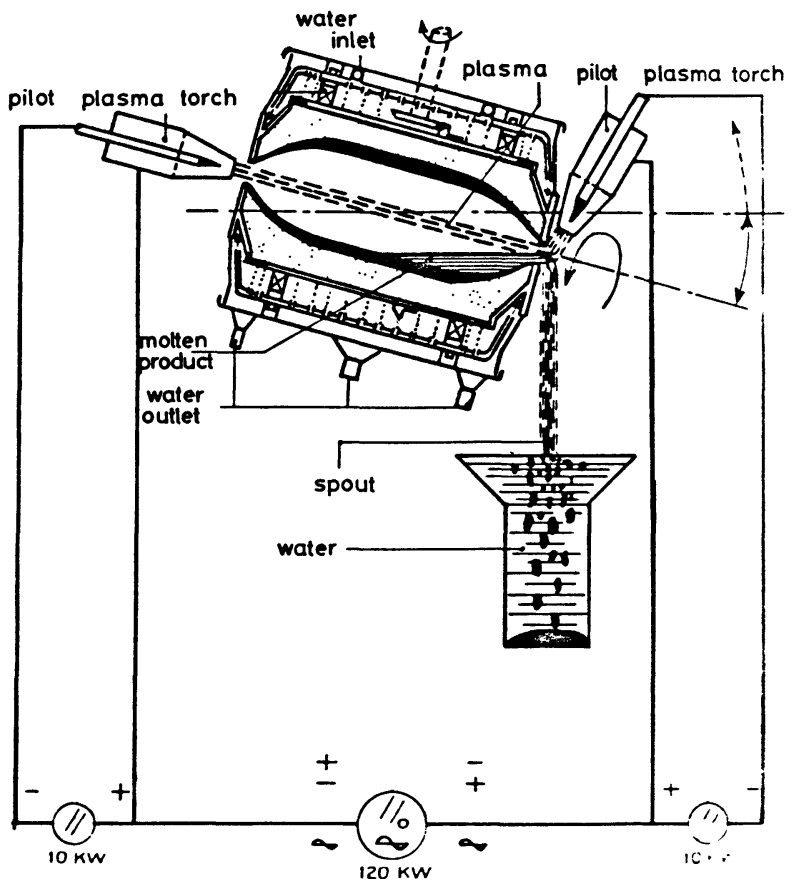

Fig. 8. - Four à plasma rotatif de $100 \mathrm{~kW}$ à axe horizontal inclinable.

[A $100 \mathrm{~kW}$ sloping rotating plasma furnace.] axe horizontal avec un chargement en matériau discontinu [37, 38] comme celui du CEA (Fig. 8) ou à axe vertical avec chargement continu du matériau [39]. Quant aux fours à parois ruisselantes, ils ont été développés par Chase [40].

d) Fours à effet d'électrode sur la charge $\left(t_{\mathrm{s}}>1 \mathrm{~s}\right)$. - Celui développé par la Bethlehem Steel [41] (Fig. 9) est une amélioration de celui de Chase, les particules venant s'accrocher sur l'anode verticale où elles forment un film ruisselant, le courant d'anode se fermant sur ce film ruisselant en augmentant notablement le transfert de chaleur à la charge. Les fours à sole [42] sont des fours conventionnels où on a remplacé les électrodes par des géné-

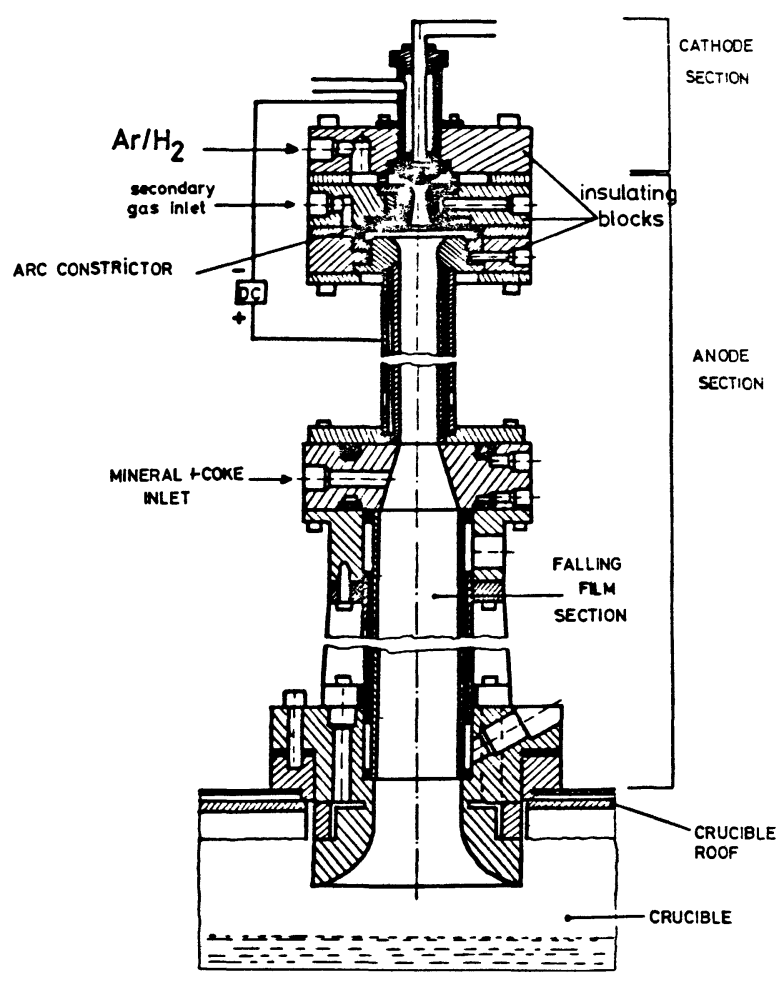

Fig. 9. - Réacteur de Bethlehem Steel de $1 \mathrm{MW}$ à film ruisselant. [Bethlehem Steel's 1 MW falling film furnace.]

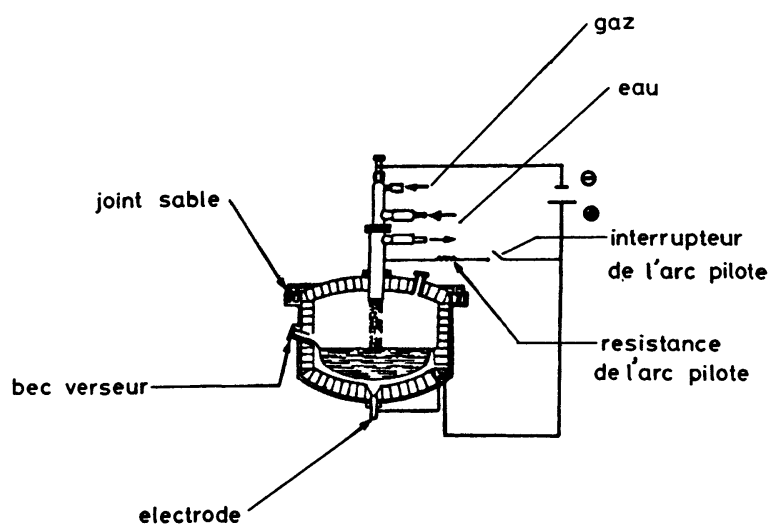

Fig. 10. - Four à plasma de la Linde Plasmarc.

[Plasma furnace of Linde Plasmarc.] 
rateurs à plasma en fermant le circuit sur la charge (Fig. 10); ils sont actuellement développés à une échelle de $10 \mathrm{MW}$ pour la métallurgie [43].

2.2 .3 Les générateurs radio-fréquence $(1$ à $20 \mathrm{MHz}$ ). - Lorsque la fréquence du courant d'alimentation est augmentée au-delà d'une fréquence critique, les ions n'atteignent plus les électrodes et leur accumulation augmente le champ électrique et par conséquent le premier coefficient d'ionisation de Townsend et l'établissement de la décharge est plus facile. Le mouvement des électrons est défini par leur mobilité qui régit la décharge. Les électrodes peuvent alors être disposées à l'extérieur de l'enceinte ce qui limite considérablement la pollution, l'enceinte étant en matériau non conducteur (quartz, nitrure de bore ...).

Ces générateurs, fonctionnant à une fréquence de 1 à $20 \mathrm{MHz}$, peuvent être utilisés avec des gaz agressifs tels que $\mathrm{O}_{2}, \mathrm{Cl}_{2}, \mathrm{UF}_{6}[44,45]$; la durée de vie des tubes HF est de l'ordre de 2 à $3000 \mathrm{~h}$ et les puissances peuvent atteindre $1 \mathrm{MW}$, les générateurs industriels ne permettant cependant pas de transférer plus de 40 à $60 \%$ de l'énergie au plasma et leur coût est 3 à 4 fois plus élevé que celui des sources d'arc (continu ou alternatif) [14].

Les générateurs les plus développés sont ceux à couplage inductif (Fig. 11 [46]). La puissance minimale et la fréquence correspondante pour que la décharge soit auto-entretenue sont fonction de la nature du gaz (dans la majorité des cas, il est nécessaire de conserver

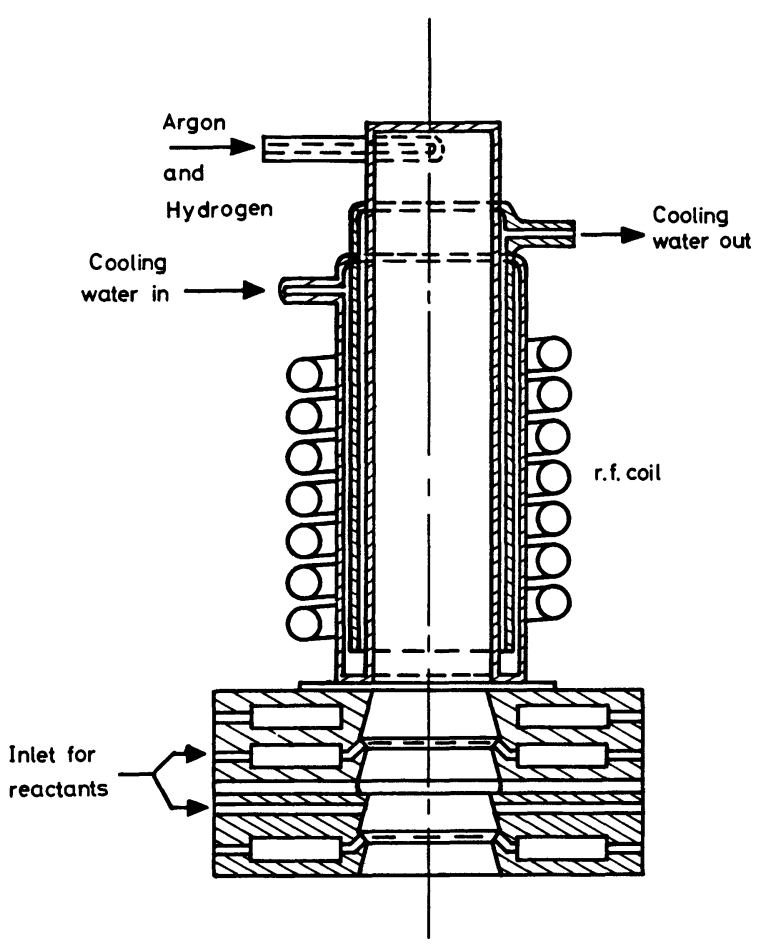

Fig. 11. - Générateur de $30 \mathrm{~kW}$ radio-fréquence à couplage selfique.

[A $30 \mathrm{~kW}$ radio-frequency-induced plasma reactor.] un certain débit d'argon), de sa pression et des caractéristiques géométriques du réacteur [47]. Lorsque la fréquence passe du $\mathrm{MHz}$ au $\mathrm{kHz}$ pour un gaz donné, la puissance minimale croît rapidement à plusieurs centaines de $\mathrm{kW}$. Les calculs relatifs aux générateurs HF sont complexes [48]. La densité d'énergie y est plus faible que pour les générateurs à arc et les vitesses d'écoulements de quelques dizaines de $\mathrm{m} / \mathrm{s}$. Les générateurs à couplage capacitifs sont encore mal connus et leur rendement assez faible du fait d'un glissement de phase entre le courant des électrodes et la décharge. En contre-partie, la puissance minimale nécessaire pour l'entretien de la décharge est beaucoup plus faible que pour le couplage selfique à fréquence égale [48].

2.2.4 Les décharges micro-ondes. - Les décharges micro-ondes permettent d'excellentes concentrations du champ électrique à l'intérieur de la cavité et, du fait de la faible valeur de la longueur d'onde, le diamètre du plasma peut être réduit à moins de un centimètre. Le champ électrique avec une fréquence $\omega$ pénètre dans le plasma si la densité des électrons y est inférieure à celle correspondant à la résonance plasma [50]. Par conséquent les fréquences utilisées sont dans la gamme $200-3000 \mathrm{MHz}$, le plasma étant produit avec une cavité résonnante [51]. Une nouvelle méthode de production des plasmas microondes est d'ailleurs apparue voici trois ans et elle a permis la production de longues colonnes de plasma par propagation d'une onde de surface [52]. Par exemple à $915 \mathrm{MHz}$ dans l'argon à la pression atmosphérique, il est possible d'obtenir une colonne de plasma de $50 \mathrm{~cm}$ de longueur avec une puissance de $700 \mathrm{~W}$ et un diamètre de la colonne d'environ $1 \mathrm{~mm}$.

2.2.5 Décharges hors équilibre (basse pression ou champ élevé). - Dans ces décharges, lorsque la pression et le courant ne sont pas trop faibles (plasma de colonne positive), les électrons sont le principal vecteur d'énergie et les fréquences de gain d'énergie sont fonction du rapport $E / p$ (en $\mathrm{V} / \mathrm{cm} /$ torr) et les fréquences de perte du rayon réduit $R p$ [53]. Par contre, avec les champs élevés $E / p>50-100$, le mouvement des électrons est du type bouffée. C'est le cas par exemple des décharges couronnes comme celles utilisées dans les ozoniseurs $(E / p>38 \mathrm{~V} / \mathrm{cm} /$ torr $)$ tel que celui représenté sur la figure 12 où les électrodes sont alimentées par des impulsions rectangulaires de $60 \mathrm{kV}$ avec une fréquence de répétition de $50 \mathrm{~Hz}$ [54] (le diélectrique solide est en verre et l'espace de décharge variable de 2 à $12 \mathrm{~mm}$ ).

Avec des champs moyens $(E<E / p<60)$ le phénomène principal est la perte d'énergie des électrons par collision inélastique. Ceci permet la création d'espèces actives : molécules excitées, radicaux libres, métastables, l'énergie des particules lourdes restant cependant faible en moyenne $(300<T g<1500 \mathrm{~K})$ ce qui permet d'obtenir des réactions de chimie 


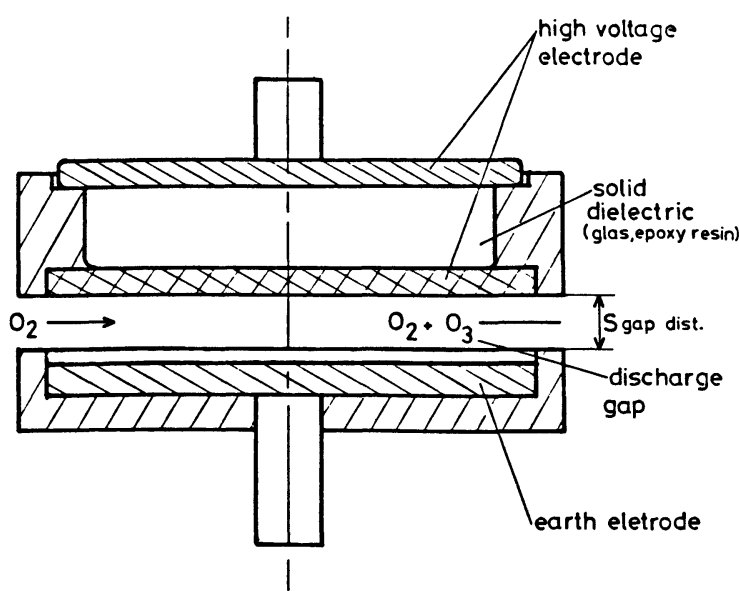

Fig. 12. - Ozonizeur.

[Ozonizer.]

organique. Les décharges luminescentes à des pressions comprises entre $10^{-1}$ et 100 torr sont très utilisées industriellement tant en courant continu que RF pour la réalisation de couches minces par plasma réactif. Par exemple le générateur de la figure 13 [55] est un système à symétrie cylindrique avec des plaques parallèles et un écoulement radial.

Le gaz entre dans la chambre de façon co-axiale, il s'écoule vers les parois sous la plaque support et ensuite revient vers le centre de la chambre au-dessus de la plaque support avant de ressortir le long de l'axe de la chambre. La décharge luminescente est encore du type radio-fréquence et elle est établie entre la plaque supérieure et la plaque support centrale maintenue à la masse. Cette plaque support est chauffée par une plaque chauffante. On peut ainsi atteindre des températures de $400^{\circ} \mathrm{C}$. L'avantage

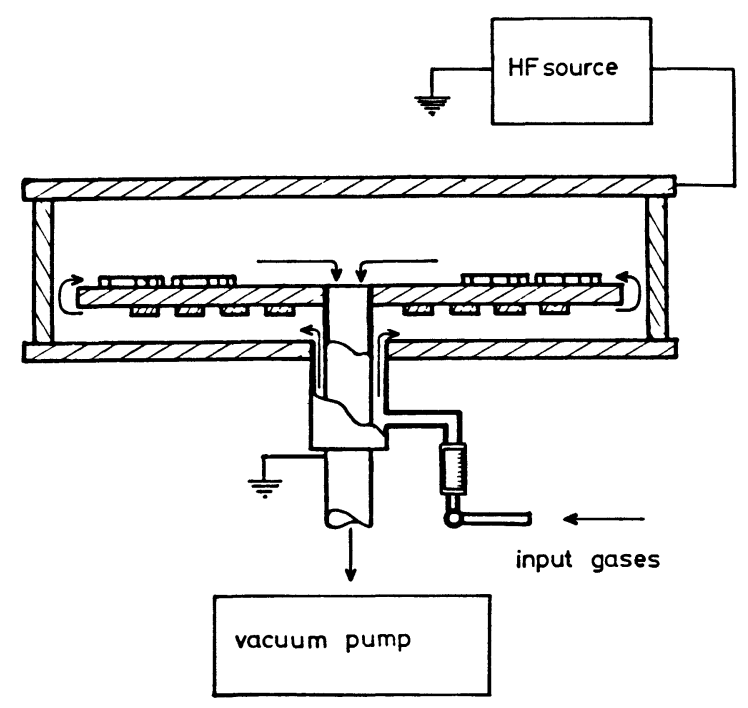

Fig. 13. - Réacteur à décharge luminescente avec écoulement radial.

[Glow discharge radial flow reactor.] de ce dispositif est de permettre le réglage des conditions d'opération de telle sorte que le gradient radial d'un processus électronique puisse pratiquement être égal à zéro. La densité des électrons contrôlée par diffusion produit une concentration élevée de ceux-ci au centre du réacteur favorisant ainsi la décomposition du gaz et une vitesse de dépôt plus grande au centre.

Des réacteurs de ce type sont également utilisés pour la gravure sous plasma (etching) en veillant en particulier à ce que le débit de gaz et le champ RF soient uniformes. On trouvera par exemple dans les références [56-59] la description de divers réacteurs utilisés dans l'industrie pour la fabrication de composante semi-conducteurs, générateurs de deux types : tunnel et plaques parallèles.

Des décharges basse pression ( $10^{-2}$ torr) sont enfin utilisées pour des techniques de dépôts telle que l'ion plating suivant le schéma représenté sur la figure 14 .

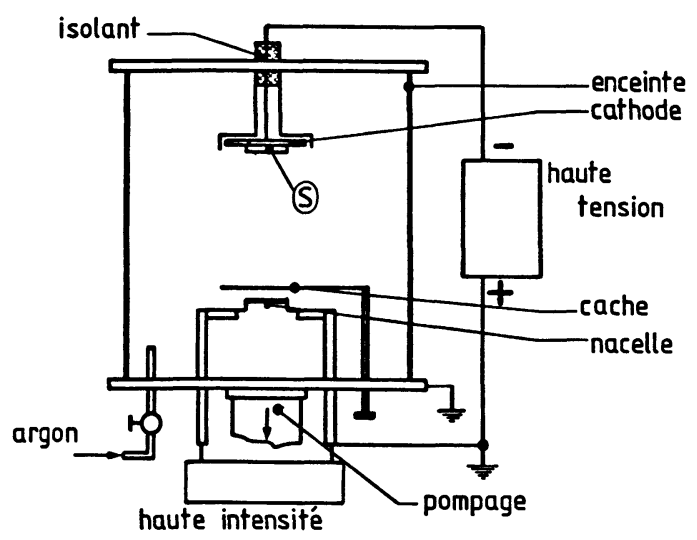

Fig. 14. - Schéma de principe de l'ion-plating.

[Principle of a reactor for ion plating.]

Le rôle de la décharge lorsque l'on évapore le produit déposé dans la nacelle est encore mal compris [60-62], mais on peut admettre qu'il est double :

- production de particules énergétiques

- production de particules excitées rendant le plasma très réactif et autorisant le dépôt d'oxydes, nitrures, carbures... formés à partir du métal évaporé.

3. Les réactions chimiques en plasma. - 3.1 LE RÉACTEUR. - Un réacteur de chimie en plasma est un tout qui doit être adapté à la réaction désirée mais que l'on divise en trois parties pour des questions de commodité de raisonnement : génération du plasma, réactions chimiques, trempe.

La figure 15 donne un schéma théorique de ces trois séquences, leur ordre possible ainsi que leur rôle [63]. Un des principaux problèmes est celui du mélange des réactifs avec le plasma qui, conformément au schéma, peut se faire avant ou après le générateur.

Le mélange des réactifs avant le générateur permet, pour les plasmas thermiques, d'éviter tous les pro- 


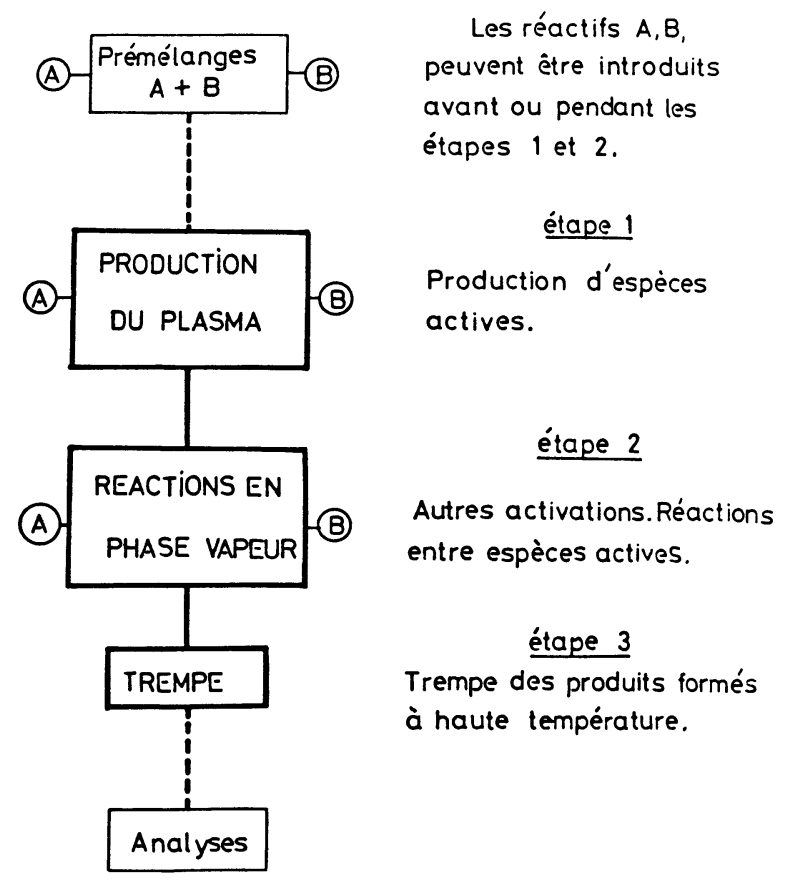

Fig. 15. - Schéma synoptique d'une réaction en chimie des plasmas.

[A plasma chemistry reaction.]

blèmes liés à l'introduction d'un produit froid solide dans le plasma (à condition toutefois que les électrodes le permettent, cf. $\S 2.2 .1$ et 2.2.2). Il permet également que les produits à traiter reçoivent une part importante (jusqu'à $60 \%$ ) de l'énergie électrique fournie. Toutefois, il n'est pas toujours bon d'exposer l'ensemble des réactifs au plasma et il est par exemple, important pour un bon rendement de synthèse des oxydes d'azote en plasma d'arc, de dissocier l'azote seul dans le plasma et d'introduire ensuite l'oxygène froid [64].

L'introduction des réactifs après la production du plasma présente certaines difficultés pour les plasmas thermiques (très visqueux) et il est difficile du fait des gradients de température et de concentration, que tous les réactifs subissent le même traitement. Enfin l'énergie transférée est faible (moins de $10 \%$ à des particules solides par exemple). De plus l'introduction de réactifs froids et non excités modifie totalement les conditions d'équilibre (ou de déséquilibre) du plasma.

Les processus sélectifs qui ont un caractère absolument fondamental au niveau des plasmas froids, jouent également un rôle important dans les plasmas thermiques. Dans ces derniers, les modes d'excitation sont souvent plus divers et ont généralement des cinétiques très différentes puisque situés à des températures et pressions beaucoup plus élevées.

La rapidité de l'excitation du gaz plasma dans la décharge joue un très grand rôle : l'importance des vitesses de chauffage des gaz dans un plasma d'arc (jusqu'à $10^{9} \mathrm{~K} / \mathrm{s}$ ) modifie totalement l'équilibre et donc la composition du plasma, améliorant sensiblement la production des oxydes d'azote [63] par exemple; il en va de même pour la production de l'ozone où le rendement est amélioré pour des pulses submicroniques [65].

La trempe des produits obtenus joue également un rôle prépondérant, conditionnant toute la synthèse dans certains cas. On admet que les deux rôles essentiels de la trempe sont [66] :

- de retirer l'excès d'énergie des nouvelles espèces excitées qui sont formées (stabilisation),

- de prévenir les réactions inverses qui détruisent les nouveaux produits formés.

La loi de trempe $T=f(t)$ ainsi que le moment où doit commencer la trempe jouent également un rôle capital : un retard de $2 \times 10^{-3}$ s réduit la conversion de l'acétylène de $15,5 \%$ à $10 \%$ et la diminution de la vitesse de trempe de $10^{8}$ à $10^{7} \mathrm{~K} / \mathrm{s}$ réduit la conversion de $\mathrm{N}_{2}$ en NO de 9,6 à $6,4 \%$ [67].

Des trempes inférieures à $10^{5} \mathrm{~K} / \mathrm{s}$ n'ont pratiquement aucun effet. De telles vitesses sont obtenues [66] par détente dans des tuyères de Laval, par injection de gaz froids, pulvérisation de liquides, parois froides...

Il faut enfin signaler que les phénomènes de catalyse, encore non maîtrisés en plasma, peuvent jouer un très grand rôle notamment pour la catalyse hétérogène [68].

3.2 L'UTILISATION DES RÉACTEURS DE CHIMIE DES PLASMAS. - Il est important de bien distinguer entre les réactions en phase gazeuse et les réactions en phase hétérogène.

3.2.1 Réactions en phase gazeuse. - Ces réactions sont extrêmement rapides avec des temps de l'ordre de $10^{-6} \mathrm{~s}$ [66]. Elles permettent suivant les conditions de température du plasma, les conditions d'équilibre et la trempe, d'obtenir diverses molécules :

- des molécules simples comme $\mathrm{NO}$ ou $\mathrm{C}_{2} \mathrm{H}_{2}$ à partir de plasma thermique, la trempe étant pratiquement le point essentiel,

- des molécules plus fragiles telles que l'hydrazine, $\mathrm{N}_{2} \mathrm{O}$ à partir d'excitation sélective, notamment par les métastables, dans des plasmas hors équilibre et dont la température des particules lourdes est faible,

- des réactions organiques telles que des isomérisations, des réactions d'insertion (azote, oxygène, monoxyde de carbone ou de soufre), des réactions d'élimination (hydrogène pour produire des molécules insaturées), des réactions de décarbonylation, des dimérisations.

3.2.2 Réactions en phase hétérogène. - Le point clef devient alors le transfert d'enthalpie plasmaparticule ou plasma-solide et l'on peut avoir toute une série de phénomènes physiques ou chimiques d'autant que pratiquement le plasma peut fondre, vaporiser et décomposer n'importe quel matériau : 
- la fusion de matériaux : de métaux pour les purifier par évaporation des matières les plus volatiles par exemple, de céramiques pour les densifier,

- la fusion de particules que l'on laisse refroidir lentement afin d'obtenir des particules sphériques,

- la vaporisation de matériaux et la recondensation des vapeurs avec ou sans réaction préalable ce qui permet d'obtenir des poudres submicroniques,

- la projection de divers matériaux qui sont fondus en plasma et projetés à grande vitesse sur un substrat ce qui permet d'obtenir des revêtements protecteurs même avec des matériaux céramiques très réfractaires,

- la synthèse de nitrures, carbures, borures à partir de métaux ou d'oxydes,

- la métallurgie extractive permettant de passer du minerai au métal en une seule opération.

Il faut cependant souligner que les réactions plasma-solide sont contrôlées par les phénomènes de diffusion et donc ont des temps caractéristiques de l'ordre de $10^{-2}$ à $10^{+1} \mathrm{~s}$ beaucoup plus lents qu'en phase gazeuse [66].

4. Les traitements de surface en plasma hors d'équilibre. - 4.1 INTRODUCTION. - Depuis une vingtaine d'années, un intérêt grandissant se manifeste pour l'utilisation des plasmas afin de modifier la structure et la composition de la surface des matériaux solides. Cet intérêt est dû d'une part à la possibilité de produire certaines modifications que l'on ne peut obtenir autrement et d'autre part à la facilité de contrôler le degré de modification. De plus, ces techniques plasma peuvent très souvent se substituer à des techniques classiques très polluantes.

Lorsque la pression est très basse, il est généralement admis que les diverses interactions, au voisinage de la paroi, peuvent être décrites par une superposition d'effets différents sans processus synergétiques. Les interactions à prendre en compte sont essentiellement celles entre les ions incidents (à faible énergie) et les atomes de la surface : diffusion, réflexion, sputtering, désorption des gaz absorbés, rupture des énergies de liaison...

Dès lors que la pression augmente il convient de prendre en compte l'interaction des ions avec les atomes du gaz qui peuvent atteindre la surface avec une grande énergie et participer à l'interaction ainsi que la formation de radicaux ou d'espèces excitées (métastables notamment) qui peuvent provoquer d'importantes modifications chimiques et physiques de la surface. De plus ces modifications sont très souvent conditionnées par les phénomènes de chimisorption qui peuvent être considérablement augmentés en présence d'ions ou d'électrons [68].

L'interaction physique du solide et du plasma [69] est soit une interaction permanente (le solide est fixé en une zone de la décharge), soit une interaction transitoire (particule passant dans le plasma). Les interactions de type transitoire sont généralement trop brèves pour qu'il y ait des modifications importantes de la surface de la particule avec un plasma basse pression et ce type de traitement est généralement utilisé avec des plasmas thermiques pour la fusion, la sphéroïdisation, ou la vaporisation des particules. Lorsque le solide est fixé dans une zone de.la décharge, le fait qu'il soit isolé (potentiel flottant) ou non modifie non seulement le rapport flux d'électrons/flux d'ions qu'il reçoit, mais aussi l'énergie cinétique des ions. Par exemple, pour une surface à un potentiel flottant par rapport au potentiel du plasma, les ions arrivent à la surface avec une énergie qui peut atteindre $5 \mathrm{~V}$ dissipée essentiellement sous forme d'effets physiques tels que l'ablation ou une charge électrostatique. Par contre si la surface sert de cathode, les ions sont accélérés dans la zone de chute cathodique, peuvent l'atteindre avec des centaines de volts d'énergie entraînant une importante élévation de température et le sputtering.

On conçoit donc que l'environnement du plasma est très complexe et qu'il est difficile de définir les paramètres importants, d'autant que le flux et l'énergie des électrons ou des ions frappant la surface, les espèces et la concentration des neutres excités ou non et des radicaux y arrivant, les espèces chimisorbées... sont souvent inconnus. Enfin agir sur un paramètre macroscopique de l'expérience modifie souvent les divers paramètres microscopiques et les résultats sont difficiles à interpréter.

L'énergie va être transférée [69] du plasma au solide par le biais :

- du rayonnement

- du flux de neutres excités ou non

- du flux de particules chargées

et être dissipée à l'intérieur du solide sous forme de nombreux processus physiques et chimiques entraînant des modifications des propriétés de surface.

Le rayonnement intervient surtout pour les polymères : l'absorption du rayonnement IR se traduit par un échauffement du matériau susceptible de le dégrader, le rayonnement visible est faiblement absorbé et ne se traduit par aucune modification chimique, le rayonnement UV est fortement absorbé et produit des radicaux qui sont des sites actifs susceptibles de réagir avec les composants du plasma.

Les particules neutres bombardent la surface et la chauffent essentiellement par dissipation de l'énergie cinétique et vibrationnelle. Pour les polymères, l'énergie de dissociation des radicaux libres est dissipée à la surface sous forme de réactions chimiques : abstraction, addition, oxydation... et de dissipation thermique. Pour les surfaces non organiques, l'énergie de dissociation est dissipée sous forme de réactions de surface par absorption. Les métastables qui ne peuvent dissiper leur énergie que par collision produisent des radicaux libres pour les polymères car leur énergie est généralement supérieure à celle de dissociation des polymères. 
Les ions, comme nous l'avons vu, suivant leur énergie d'impact produisent soit l'ablation de la surface, soit le sputtering et le chauffage de celle-ci. Les ions produits par ionisation dissociative, et qui bombardent aussi la surface, conduisent à des réactions similaires à celles obtenues par dissociation des particules. L'énergie d'ionisation est dissipée par neutralisation à la surface et se retrouve sous forme d'énergie thermique, pour la production de radicaux libres de polymères.

Comme l'a souligné Winters [68] un des processus les plus importants est le transfert chimique à la surface, phénomène où l'on peut distinguer les étapes suivantes :

— diffusion du réactif $\mathrm{A}$ à la surface solide (s)

- adsorption du réactif $\mathrm{A}$

- réaction du type $\mathrm{S}(\mathrm{s})+\mathrm{A}(\mathrm{g}) \rightarrow \mathrm{B}(\mathrm{g})$

- désorption du produit de réaction $B$

- diffusion du produit $B$ à l'extérieur de la surface.

Ce processus, hors équilibre chimique du fait $\mathrm{du}$ plasma, permet d'expliquer par exemple que le réactif $\mathrm{A}$ soit en concentration supérieure à celle qu'il aurait à l'équilibre et que le produit de réaction $B$ soit en concentration inférieure, ce qui entraîne le phénomène de gravure.

Réciproquement si le produit de réaction $B$ est en excès, il y a un phénomène de dépôt. La première étude théorique de ce phénomène, confirmée d'ailleurs par l'expérience, a été développée par Zarowin [70] avec une surface d'or dans un plasma de chlore. Cependant ces phénomènes de chimisorption restent encore mal connus, le seul point dont on soit sûr est qu'ils sont considérablement augmentés du fait de la présence d'ions ou d'électrons. Winters par exemple [68] a montré que $\mathrm{XeF}_{2}$ seul en présence de la paroi $\left(\mathrm{Si}\right.$ ou $\mathrm{SiO}_{2}$ ) n'entraîne pas de phénomène de gravure alors qu'en présence d'électrons pour $\mathrm{Si}$, ou d'ions $\mathrm{Ar}^{+}$pour $\mathrm{SiO}_{2}$, les vitesses de gravure peuvent atteindre $200 \AA / \mathrm{min}$.

Enfin comme l'a montré Rapakoulias [71] ces phénomènes de chimisorption peuvent modifier totalement les réactions chimiques. Par exemple une surface de molybdène permet par chimisorption de casser les molécules $\mathrm{N}_{2}\left(\mathrm{~A}^{3} \Sigma^{+}\right)$en deux atomes $\mathrm{N}-\mathrm{N}$ susceptibles alors de réagir avec du méthane ou des radicaux pour former une molécule $\mathrm{HCN}$. On peut ainsi doubler, en présence d'une grille en molybdène, le taux de conversion en HCN dans un plasma d'azote produit par une décharge haute fréquence et trempé par une injection de méthane froid. Ces phénomènes de catalyse hétérogène ouvrent ainsi une voie très prometteuse en chimie des plasmas.

4.2 LES TECHNIQUES DE DÉPÔT PAR PULVÉRISATION CATHODIQUE ET ION PLATING. - Dans ces procédés de dépôts sous vide le transfert du corps à déposer sur le substrat que l'on veut recouvrir se fait sous vide ou sous une pression réduite. Les dépôts peuvent s'effectuer de plusieurs manières par le transfert :
- soit de molécules et d'atomes : évaporation thermique et pulvérisation cathodique, d'ions,

- soit d'un mélange d'ions et d'atomes : ion plating.

Les qualités que l'on demande à un dépôt dépendent de son utilisation future. Ainsi en électronique on cherche en premier lieu à réaliser des dépôts de grande pureté ayant une structure parfaitement déterminée. En traitements de surfaces, au contraire, on cherche à obtenir :

- une grande vitesse de dépôt,

- une excellente adhérence du dépôt sur le corps à recouvrir,

- un très bon pouvoir de recouvrement.

Une méthode donnée ne permet pas d'obtenir des dépôts répondant à tous ces critères.

4.2.1 Pulvérisation cathodique. - Elle a été développée à partir de 1953 [72]. Les atomes ou molécules du corps à déposer sont éjectés d'une cible bombardée par un flux de particules énergétiques, des ions positifs en général. Ces ions sont produits dans une décharge luminescente qui s'établit entre deux électrodes : la cathode, ou cible, qui est constituée par le matériau à pulvériser, l'anode qui porte le substrat [73]. La décharge est amorcée en introduisant dans l'enceinte un gaz neutre au réactif. On distingue le procédé diode en régime continu ou haute fréquence et le procédé triode.

La pulvérisation cathodique permet de déposer pratiquement tous les matériaux. Elle est particulièrement appropriée dans les cas suivants : matériaux réfractaires difficiles à évaporer, alliages (les dépôts ont la même composition que le matériau d'origine), matériaux composés (nitrures, carbures, oxydes), couches conductrices, résistantes, semi-conductrices, diélectriques, ferro-électriques, couches anti-usure, couches anti-corrosion...

Actuellement, on tente d'améliorer les vitesses de dépôts $\left(1\right.$ à $\left.10 \mu \mathrm{m} \mathrm{h}^{-1}\right)$ en utilisant de nouveaux systèmes de pulvérisation (magnetron [74]).

4.2.2 L'ion-plating. - Cette technique préparée en 1963 par Mattox s'est développée récemment. Son principe est simple : il s'agit d'une évaporation réalisée en présence d'une décharge électrique. Le corps à déposer (Fig. 14) est placé dans une nacelle et évaporé par effet Joule ou par faisceau d'électrons et les substrats sur lesquels on veut effectuer les dépôts, sont placés sous la cathode $\mathrm{C}$ qui peut être portée à un potentiel continu ou haute fréquence (suivant que le substrat est conducteur ou non) pouvant varier de 0 à $5000 \mathrm{~V}$ environ. L'introduction d'un gaz à une pression de l'ordre de $10^{-2}$ torrs permet d'établir la décharge entre la cathode et l'enceinte à la masse. Cette décharge permet d'obtenir des particules énergétiques et des particules excitées 
dans le plasma. Au début en présence d'un gaz rare et sans évaporation, les ions du gaz support pulvérisent le substrat et permettent de le décaper sur une profondeur de plusieurs dizaines d'angströms et de le débarasser de toutes ses impuretés. Ensuite dès que commence l'évaporation les ions sont soit ceux du gaz support, soit ceux du corps évaporé, soit ceux du substrat. Le libre parcours moyen étant faible (pour $p \sim 10^{-2}$ torr, $\lambda \sim 5 \mathrm{~mm}$ ) une partie importante des ions et des atomes du substrat retombe sur ce dernier en se mélangeant intimement avec les particules que l'on veut déporer et les ions du corps à déposer ayant acquis une énergie importante dans la chute de potentiel cathodique pénètrent plus ou moins dans le substrat. Il y a donc réalisation d'une interface entre le substrat et le dépôt où se mélangent intimement les molécules du substrat avec celles du corps à déposer. Ceci explique que l'on obtienne des dépôts très adhérents. Lorsque le dépôt a une certaine épaisseur, le substrat n'est plus atteint et les ions du gaz support pulvérisent le dépôt ce qui permet d'obtenir une porosité très faible [75]. Si l'on introduit avec le gaz rare (argon en général) un gaz réactif : azote, oxygène, méthane..., il se crée alors dans le plasma une grande variété d'espèces réactives qui peuvent former de nouveaux produits avec les atomes évaporés : nitrures, carbures par exemple, qui viennent alors se déposer suivant le processus décrit. En résumé, cette technique est donc caractérisée par :

- une grande vitesse de dépôt (en moyenne $100 \mu \mathrm{m} \mathrm{h}^{-1}$ ),

- une très bonne adhérence (réalisation d'une interface où se mélangent intimement substrat et dépôt, ajoutée à la pénétration des ions),

- un dépôt sur toutes les faces du substrat quelque soit sa forme géométrique sans que la répartition soit toutefois homogène [76, 77],

— une porosité des dépôts très faible [75].

4.3 Les tRAITEMENTS DE SURFACE. - On utilise généralement des décharges luminescentes (cf. Fig. 13) pour :

4.3.1 La nitruration et la carburation des métaux. Lorsque les atomes d'azote réagissent avec les atomes métalliques à l'intérieur du métal, il y a formation de nitrures et une augmentation sensible de la dureté superficielle du métal ainsi traité. L'introduction de l'azote atomique dans le métal est obtenue soit par absorption d'espèces neutres, soit par bombardement avec des ions [78, 79] et il est même possible de contrôler la nature du nitrure formé $\left(\mathrm{Fe}_{4} \mathrm{~N}(\gamma)\right.$, $\left.\mathrm{Fe}_{2} \mathrm{~N}(\sigma) \ldots\right)$.

Il est également possible de produire des cristaux de nitrure à partir du traitement en plasma de mélanges chlorures-azote : $\mathrm{AlN}, \mathrm{TiN}$ [80], $\mathrm{Si}_{3} \mathrm{~N}_{4}$ [81]. La carburation et la carbonitruration peuvent s'obtenir à partir des mêmes principes à condition que le réacteur soit muni d'un dispositif de trempe. Par exemple une carbonitruration à $860^{\circ} \mathrm{C}$ sur une épaisseur de $0,6 \mathrm{~mm}$ demande $1 \mathrm{~h}$ contre 4 par voie thermique.

4.3.2 La modification des propriétés des surfaces polymériques. - Le traitement en plasma de surfaces polymériques modifie considérablement leurs propriétés de mouillabilité en ajoutant des groupes fonctionnels oxygène et non oxygène à la surface, les premiers entraînant des changements plus importants de la mouillabilité que les seconds [82], les temps d'oxydation en plasmas sont très brefs (de l'ordre de quelques secondes [83]). On trouvera par exemple dans [84] une étude détaillée de ces phénomènes qui ont de nombreuses applications dans l'emballage, l'électronique, la construction et l'industrie du vêtement en permettant notamment l'impression directe sur la surface polymérique sans changement des propriétés intrinsèques du polymère. Ces modifications permettent également le collage ou la fixation de matériaux divers sur les polymères.

4.3.3 Le dépôt de films organiques. - Les films diélectriques minces sont utilisés pour la fabrication des semi-conducteurs et leurs méthodes de dépôt par plasma en décharge luminescente se développent considérablement [85]. Par exemple pour les nitrures de silicium les dépôts peuvent être obtenus à l'ambiante contre $800^{\circ} \mathrm{C}$ pour les dépôts par évaporationréaction chimique. Pour les matériaux à base de silicium généralement utilisés la source est le silane $\left(\mathrm{SiH}_{4}\right)$ qui en présence d'oxygène (moins de $7 \%$ ) permet d'obtenir des dépôts de silice $\mathrm{SiO}_{2}$, qui peut se polymériser $\left(\mathrm{SiH}_{n}\right)$ ou former des nitrures [86].

Ces dépôts sont d'autant plus adhérents que la décharge plasma, dans l'argon par exemple, avant l'introduction des gaz réactifs, permet un nettoyage excellent de la surface. Les propriétés des dépôts dépendent de la composition des gaz, du temps de résidence, de la puissance de la décharge... [85]

4.3.4 La gravure en plasma. - Les techniques plasma de nettoyage, de gravure ou d'enlèvement de masques présentent un intérêt croissant pour la technologie des semi-conducteurs [87, 88] d'autant que ces techniques permettent des gravures de circuits très précises et qu'elles réduisent très sensiblement le nombre d'opérations. Actuellement l'effort porte sur des techniques de gravure sélective permettant de ne pas attaquer la couche inférieure.

Le dessin à obtenir est délimité par une couche de résine photosensible et l'attaque de la surface est effectuée par des espèces actives du fluor obtenues par la décomposition en plasma, du fait des ions ou des électrons, de gaz tels que $\mathrm{CF}_{4}$ suivant par exemple les réactions :

$$
\begin{aligned}
& \mathrm{CF}_{4} \rightarrow \mathrm{C}+4 \mathrm{~F}^{*} \\
& 4 \mathrm{~F}^{*}+\mathrm{SiO}_{2} \rightarrow \mathrm{SiF}_{4}+2 \mathrm{O} .
\end{aligned}
$$

Il convient cependant de noter que les controverses à ce sujet sont encore nombreuses et le rôle 
des espèces chargées, bien que mal compris, est très important [68]. La couche de résine est ensuite enlevée par action de l'oxygène atomique produit en plasma [89].

4.3.5 Les dépôts de films organiques. - Une grande variété de composés peuvent donner des dépôts solides par polymérisation en plasma : aussi bien les monomères classiques que ceux qui résistent à la polymérisation comme les aromatiques. Les produits de base les plus couramment utilisés sont le diméthylsiloxane, les silanes, le cyclobenzène, le styrène, le toluène, le xylène, le benzène [90]. Il semble que la polymérisation s'effectue convenablement si le monomère contient une liaison carbone-hydrogène, une double liaison carbone-carbone ou un noyau aromatique. On peut également faire varier plus ou moins continûment la composition des dépôts en modifiant le monomère pendant l'opération (par exemple passage du polyéthylène au polytétrafluorétylène).

Les films obtenus sont : exempts de trous, fortement réticulés, stables thermiquement et très faiblement solubles pour des épaisseurs comprises entre quelques centaines d'angströms et quelques microns. Par contre ils ont une forte concentration de spins non apairés et ils présentent une oxydation rapide de leur surface. Ils ont en particulier des propriétés électriques intéressantes : permittivités relatives entre 2,5 et 4 , rigidité diélectrique supérieure à $10^{6} \mathrm{~V} / \mathrm{cm}$; résistivité transversale entre $10^{12}$ et $10^{14} \Omega \mathrm{m}$. Ces films sont utilisés pour la réalisation de microdensateurs à grande capacité spécifique ou pour la passivation des circuits (couches de styrène à la place de la silice [90]).

5. Applications des plasmas. - Dans ce paragraphe nous nous proposons de passer sommairement en revue les applications des plasmas déjà industrialisées et celles qui pourraient l'être à moyen terme. Toutefois avant d'examiner ces applications dans le détail nous tenterons de situer l'intérêt des procédés plasma utilisant l'énergie électrique dans la conjoncture énergétique actuelle (intérêt justifié pour les productions à fort tonnage). Nous passerons ensuite aux applications proprement dites en les séparant en production de fort tonnage (généralement à faible valeur ajoutée) pour lesquelles le coût de l'énergie est important et en production à forte valeur ajoutée pour lesquelles le coût de l'énergie est relativement moins élevé.

5. 1 Problème ÉNERgétiQue. - La diminution rapìde des réserves mondiales d'hydrocarbures ou de gaz naturel et l'augmentation considérable de leur prix impliquent une réévaluation complète de leur utilisation respective en tant que source d'énergie. Compte tenu des réserves dont on dispose en énergies (cf. Tableau II de [91] et Tableau III de [92]), le développement de l'utilisation de l'électricité produite soit à partir des combustibles nucléaires, soit à partir des réserves de charbon semble être une solution logique au problème énergétique à long terme.

Par ailleurs, jusqu'à présent, les sources mondiales d'énergie et de matières premières carbonées se sont développées à partir du pétrole et du gaz naturel et le sort de la chimie lourde est apparu lié à celui de l'énergie [92].

Dans ces conditions, outre le développement de nouvelles énergies, une distinction entre "matières premières à usage énergétique et à usage chimique est-elle en train d'apparaître ? Par exemple aux U.S.A., on prévoit [93] l'augmentation croissante des besoins pétrochimiques pour la chimie lourde, alors qu'au niveau énergétique il pourrait $y$ avoir en partie substitution du nucléaire, du charbon ou d'énergies renouvelables au pétrole. Reste cependant que cette utilisation, par exemple du charbon et des schistes bitumineux, est grevée par les conditions d'extraction (par comparaison, la tonne de charbon est de $1 \$$ en Afrique du Sud contre 20 et 40 \$ en Europe [92-94]).

Ce nouvel échantillonnage des divers types d'énergie et d'utilisation des matières premières nécessite naturellement en retour que de nombreux processus chimiques à hautes températures utilisent des dispositifs de chauffage à énergie électrique ou que soient utilisées des propriétés spécifiques des plasmas hors d'équilibre pour réaliser dans des conditions avantageuses une stéréochimie très spécifique.

Enfin il faut souligner que les processus de chimie des plasmas présentent des avantages qui ne peuvent que les valoriser dans les années à venir :

- ils correspondent à une très forte concentration d'énergie qui rendent les installations très petites pour ne pas dire miniatures par rapport aux procédés classiques,

- ils peuvent être mis en route et arrêtés en quelques minutes, ce qui supprime beaucoup de contraintes (en métallurgie extractive par exemple),

- les problèmes de pollution $y$ sont beaucoup plus faciles à maîtriser.

5.2 ProcédÉS Plasma DÉJA INDUSTRIALISÉS. - Les procédés plasmas passés au stade industriel sont très divers et ils recouvrent quelques produits à fort tonnage et faible valeur ajoutée pour lesquels l'utilisation du plasma est un peu limitée mais surtout des produits à forte valeur ajoutée.

5.2.1 Produits à moyenne valeur ajoutée. - a) Sphéroïdisation. - Tout matériau susceptible de fondre (sans se décomposer ou se vaporiser) devient sphérique à la fusion sous l'action des forces de tension superficielles, forme conservée à la solidification. La fusion en plasma conduit également à la purification par évaporation des impuretés. Les grands procédés sont :

- la production [95] de sphères de zircone (1 $000 \mathrm{t} / \mathrm{an})$ par traitement dans un four du type de celui de la figure 6 de zircon (la matrice de silice 
qui se forme autour de la sphère de zircone est éliminée par lavage à la soude). Cette zircone est utilisée pour le frittage de céramiques ayant une bonne tenue aux chocs thermiques, pour la fabrication de certains verres ou de zirconium [96, 97],

- la production, pour la photocopie, de sphères de magnétite par Westinghouse [98] et Alu Suisse [99], de ferro-silicium par Rank Xerox [100].

b) Refusion des métaux. - La refusion des métaux en plasma améliore considérablement leur qualité, de plus contrairement à la fusion sous vide, les pertes par évaporation de composants intéressants comme le manganèse, le molybdène et le magnésium peuvent être très réduites. Le plasma permet également l'amélioration d'alliages contenant des espèces facilement oxydables et chimiquement actives comme le titane et l'aluminium. Ces fours fonctionnent essentiellement à l'argon, à l'argon-hydrogène ou à l'argon-azote. Outre le fait que la puissance électrique, contrairement aux fours à électrodes, est indépendante de l'état d'avancement de l'opération et qu'il y a très peu de fluctuations, l'utilisation des espèces actives du plasma permet diverses transformations chimiques que l'on ne pourrait obtenir autrement [101]. Dans tous ces fours les électrodes classiques sont remplacées par des générateurs à plasma avec ou sans superposition de puissance [101].

Le four à sole de la Linde [42] (cf. Fig. 10) a été le premier prototype. Les fours de ce type sont actuellement développés à l'échelle de $10 \mathrm{t}$ (jusqu'à $30 \mathrm{MW}$ ) en RDA, en URSS [43, 102]. Les propriétés des métaux obtenus sont celles des fours sous vide. Le gaz plasmagène est généralement l'argon et la consommation énergétique varie de 2 à $0,1 \mathrm{kWh} / \mathrm{kg}$. Il existe au Japon [103] un four chauffé à la fois par une torche à plasma et par induction pour la purification $\mathrm{du}$ titane, du chrome et la fusion des alliages de cuivre.

Des fours de refusion utilisant un plasma obtenu à partir de deux ou trois générateurs et alimentés par des lingots sont en opérations en URSS [101, 104] à l'Institut Baikov où Rykalin et ses collaborateurs ont développé des fours de 150 à $200 \mathrm{~kW}$ pour la purification des métaux. Souvent également le plasma est transféré sur la surface du lingot en fusion qui sert alors d'anode. En atmosphère d'azote on peut alors obtenir la nitruration ou la surnitruration (jusqu'à $1,7 \%$ ) des aciers et alliages, les nitrures apparaissent sous forme très dispersée dans le métal.

La ULVAC Corporation au Japon commercialise un four à faisceau plasma qui permet d'obtenir des métaux de pureté similaire à ceux obtenus à partir des fours à faisceau d'électrons mais qui exige des conditions de vide beaucoup moins rigoureuses [105].

Signalons également la four de la société Electrotherm [106] représenté sur la figure 16 et destiné à l'affinage du tantale, niobium, molybdène, à la fusion des alliages réfractaires au cobalt ou au nickel,

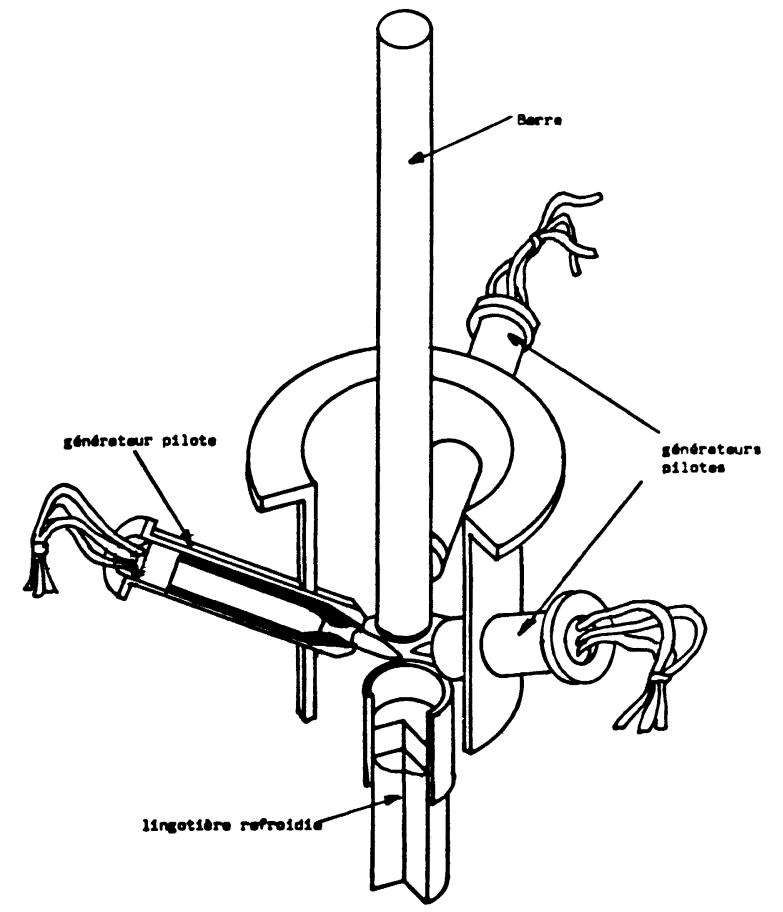

Fig. 16. - Affinage des métaux sous plasma triphasé, réacteur ARCOS.

[Metal refining with a three phase plasma reactor (ARCOS).]

des alliages de cuivre... (puissance $250 \mathrm{~kW}$, consommation $<5 \mathrm{kWh} / \mathrm{kg}$ ).

Dans ce type de four, le poste le plus important n'est pas la consommation énergétique mais la consommation en gaz des générateurs à plasma pilote (argon, hélium). Le développement dépend donc de l'utilisation de gaz moins cher mais aussi de l'augmentation de la capacité des installations (10 à $15 \mathrm{t}$ ), en effet, la consommation de gaz est pratiquement indépendante de la puissance.

c) Production des particules ultra-fines. - Des poudres ultra-fines ayant une surface spécifique très élevée et des propriétés particulières sont obtenues par vapo-condensation des réactifs. Leur industrialisation est réalisée pour les applications suivantes :

- réalisation de pigments de titane à partir de la réaction du chlorure de titane avec un plasma d'oxygène soit à haute fréquence [101] (à l'échelle de $400 \mathrm{~kW}$ en URSS), soit à arc [109] (à l'échelle de $500 \mathrm{~kW}$ par la Tioxide),

- réalisation de poudres ultra-fines de silice.

Ces poudres sont généralement obtenues à partir de la silice vaporisée en plasma (souvent en atmosphère oxydante). Les consommations énergétiques sont de l'ordre de 10 à $20 \mathrm{kWh} / \mathrm{kg}$ avec des fours à arc à l'échelle de 200 à $700 \mathrm{~kW}$ [109, 110]. Les propriétés de ces poudres (submicroniques et sphériques) sous forme de chaînes avec des radicaux $\mathrm{OH}$ dépendent en fait de l'atmosphère et de la vitesse de trempe des phases condensées. 
Enfin maintenant plus de $60 \%$ des fibres optiques de la Bell co. sont fabriquées par la technique plasma HF en effectuant la pyrolyse des chlorures de silicium et de gallium circulant dans un tube de silice. Cette technique permet simultanément le dépôt du cœur de la fibre et l'étirement du tube de silice.

d) La projection plasma. - C'est là certainement l'utilisation industrielle la plus développée des plasmas pour la réalisation d'une couche protectrice par projection de particules métalliques ou céramiques fondues. L'avantage principal de la projection par plasma sur la projection par flamme est d'une part la grande vitesse atteinte par les particules (jusqu'à $500 \mathrm{~m} / \mathrm{s}$ ) et d'autre part le fait qu'il soit possible de fondre les matériaux les plus réfractaires, compte tenu des températures atteintes dans le plasma. La projection plasma est donc particulièrement bien adaptée pour la formation de dépôts céramiques ou métalliques destinés à la protection contre l'usure, la corrosion ou comme barrière thermique ou électrique.

On trouvera de nombreux détails dans les revues générales publiées récemment sur ce sujet [111-113] ainsi que sur les matériaux que l'on peut projeter $[113,114]$ (comme pour la sphéroïdisation il suffit que le point de fusion soit éloigné de celui de vaporisation ou de dissociation).

5.2.2 Les produits à forte valeur ajoutée. - Les produits à forte valeur ajoutée produits par plasma sont essentiellement des couches minces inorganiques ou non et la gravure des circuits. Des couches minces sont en général déposées sur une pièce ou un substrat de moindre valeur pour lui conférer des propriétés de surfaces spécifiques. L'industrie s'est intéressée particulièrement à ces dépôts tant au plan de la résistance à la corrosion, aux chocs thermiques, à l'usure qu'à celui des propriétés optiques spécifiques ou de la passivation des circuits électroniques. La gravure des circuits électroniques remplace quant à elle les techniques de gravure humide. Comme nous l'avons vu les plasmas utilisés sont de faible puissance (moins de $1 \mathrm{~kW}$ ) et n'ont donc pas d'intérêt énergétique particulier, mais la valeur ajoutée au produit est très grande et le traitement plasma infiniment moins polluant que tous les traitements classiques.

a) Circuits électroniques. - La chimie des plasmas froids a trouvé une application croissante dans la technologie des circuits intégrés. Elle intervient dans deux procédés qui sont apparus depuis quelques années :

- Le dépôt chimique activé par plasma (plasma activated CVD) permet la passivation des circuits intégrés au moyen de couches de protection de $\mathrm{Si}_{3} \mathrm{~N}_{4}$ déposées à des températures inférieures à $400^{\circ} \mathrm{C}$. Cette passivation basse température est effectivement utilisée industriellement (ex. Texas Instruments). Des réacteurs sont commercialisés depuis 1976 (Applied
Materials). En France, le LETI en collaboration avec Saphymo Stel, a mis au point un réacteur de dépôt destiné à la commercialisation. Une autre application existe : la fabrication de fibres optiques d'un co-dépôt de $\mathrm{SiO}_{2}$ et $\mathrm{GeO}_{2}$ par plasma (Philips ; Aachen, RFA).

- La gravure sèche sous plasma (plasma etching) [115] par attaque sélective des matériaux $\left(\mathrm{Si}, \mathrm{SiO}_{2}, \mathrm{Si}_{3} \mathrm{~N}_{4}, \mathrm{Al}\right)$, par réaction avec un plasma réactif $\left(\mathrm{CF}_{4}\right.$ par ex.) est une alternative aux procédés humides utilisés dans la fabrication de composants électroniques. Ce procédé, qui peut être suivi dans un plasma d'oxygène par l'enlèvement des résines ayant constitué le masque ainsi que par un nettoyage préalable de surfaces, dans un plasma d'argon par exemple, réduit considérablement le nombre des opérations par rapport à la gravure humide et est intensivement utilisé par des sociétés comme IBM, Texas, Mitsubishi, IAF, LFE, IPC... notamment pour le développement des LSI (Large Scale Integrated Circuits).

b) Les traitements de surface inorganique. La pulvérisation cathodique se développe pour la préparation de couches résistantes, conductrices, semi-conductrices, diélectriques, ferro-électriques, pour l'électronique [116-119]. En mécanique elle permet le dépôt de couches dures anti-usures en alumine, oxyde de chrome, carbure de silicium, de titane, de tungstène [120]. Elle est aussi utilisée pour le dépôt de couches anti-corrosion par exemple comme l'alumine [121].

La technique d'ion plating étant toute récente, son implantation dans l'industrie est encore limitée. $\mathrm{Au}$ Japon la société ULVAC commercialise des installations pour les dépôts de carbure ou de nitrure de titane sur les outils de coupe, des dépôts de chrome sur acier ou plastique, de dépôts de cuivre ou de nickel sur tôles d'acier. Aux USA Mac Donnell utilise cette technique pour la protection des boulons par des couches d'aluminium.

c) Les traitements de surface organique. Les traitements de surfaces polymériques ont pour objectif de conférer des propriétés nouvelles à la surface : adhésivité, mouillabilité... Ces traitements s'appliquent soit aux films polymériques, soit aux fibres artificielles ou naturelles.

Ces applications visent l'encrage, le collage ou le dépôt de peinture à la surface du film polymérique [122] afin de fournir des matériaux associant les propriétés mécaniques du substrat, les propriétés non toxiques de ces matériaux pour l'emballage alimentaire, aux propriétés d'impression de la surface.

Cette technique déjà employée par l'industrie (RP cellophane) évite les traitements par solvants qui présentent, en outre, l'inconvénient de laisser en surface des produits toxiques. Elle est également employée par l'imprimerie pour l'encrage de polyéthylène [123]. 
Rappelons enfin, comme nous l'avons déjà signalé que les dépôts de films polymériques en plasma commencent à être utilisés pour la passivation de circuits électroniques et pour la fabrication industrielle de micro-condensateurs.

d) Autres applications. - La Société Jobin Yvon commercialise un appareillage de plasma HF où l'injection de quantités de produits très dilués permet de les doser par spectroscopie d'émission du fait des transferts d'excitation (200 installations déjà vendues à ce jour).

5.3 LES PROCÉDÉS POTENTIELLEMENT INDUSTRIALISABLES. - Comme nous l'avons vu jusqu'à présent, l'utilisation des plasmas dans l'industrie s'est surtout développée au niveau des procédés conduisant soit à des produits à forte valeur ajoutée, soit à moyenne valeur ajoutée et pour lesquels souvent la technique plasma n'a pas de concurrent direct eu égard à la qualité des produits obtenus. Toutefois, compte tenu de la crise énergétique et du coût croissant des hydrocarbures, on peut penser que d'ici moins d'une décennie, certains projets plasmas verront le jour pour l'obtention de produits à faible valeur ajoutée.

5.3.1 Produits à assez faible valeur ajoutée. a) Produits gazeux. - La production de l'acétylène semble être un excellent exemple du passage, puis de l'abandon, puis à nouveau du passage d'un procédé plasma au stade industriel. En effet des grands fours à plasma à l'échelle de $10 \mathrm{MW}$ ont été développés par Huels [124, 125], Hoechst, Dupont de Nemours [126] vers les années 1960 pour la production de l'acétylène à partir des hydrocarbures. Vers 1970, ils furent abandonnés du fait de la voie éthylène meilleure marché. Mais compte tenu des conditions énergétiques actuelles, AVCO [127, 128], envisage de construire un four de $10 \mathrm{MW}$ utilisant le charbon comme matière première. Les calculs économiques d'AVCO indiquent que compte tenu de l'augmentation régulière du prix des hydrocarbures, un tel procédé pourrait rapidement coûter 0,5 à 0,7 fois moins que le procédé classique. Les processus de catalyse pourraient également améliorer sensiblement les taux de conversion $[129,130]$.

Les procédés actuels de synthèse des oxydes de l'azote exigent le reforming du méthane pour produire d'abord l'hydrogène, puis l'ammoniac, et enfin par combustion catalytique les oxydes d'azote, et par la suite l'acide nitrique. L'avantage des processus plasma serait de permettre la production directe des oxydes d'azote indépendamment de l'ammoniac, et dans des proportions qui correspondent aux besoins de l'agriculture. Les nombreuses études à l'échelle du laboratoire semblent très prometteuses [131-141] (par exemple on a obtenu en plasma d'arc [140] des taux de conversion de l'azote en oxyde d'azote de $10 \%$ et une consommation énergétique de $25 \mathrm{kWh} / \mathrm{kg}$ ) on peut espérer assez rapidement descendre à $7 \mathrm{kWh} / \mathrm{kg}$ de NO produit, ce qui rendrait le procédé industriellement viable dès maintenant. En remarquant également que selon l'évolution $d u$ prix du méthane choisi, le procédé serait viable en 1985 entre 13 et $18 \mathrm{kWh} / \mathrm{kg}$. Là aussi également la catalyse semble très prometteuse [137].

- La fixation de l'azote peut aussi être opérée en plasma sous forme d'acide cyanhydrique $[142,143]$ et également de cyanogène [144].

b) Les produits solides. - L'utilisation des plasmas pour la métallurgie extractive des ferro-alliages et des métaux nobles est dès maintenant envisageable. De nombreux travaux sont d'ailleurs actuellement développés et ils ont montré en particulier que les fours à plasma de métallurgie sont capables de produire en une seule opération des produits ultra-purs avec des consommations énergétiques très compétitives entre 3 et $12 \mathrm{kWh} / \mathrm{kg}$ de produit suivant les produits. On peut citer, pour les opérations de pyrolyse ou de réduction, par exemple :

- les études de Noranda sur la molybdénite (à l'échelle de $100 \mathrm{~kW}$ ) [145-148],

- les études sur les ferro-alliages : ferro-vanadium par la Bethlehem à l'échelle de $400 \mathrm{~kW}[149,150]$ (consommation énergétique de $5,5 \mathrm{kWh} / \mathrm{kg}$ ), ferrochrome (Westinghouse [151, 152], l'Université de Toronto [153] et celle de Limoges [154] à des échelles de $100 \mathrm{~kW}$ à $1 \mathrm{MW}$ ) (consommation entre 6 et $12 \mathrm{kWh} / \mathrm{kg}$ ),

- les études de Bethlehem à l'échelle de $1 \mathrm{MW}$ sur la production du fer $[155,156]$ dont l'évaluation économique [157] semble très encourageante pour les opérations de fonderie (coût énergétique de $3 \mathrm{kWh} / \mathrm{kg}$ conduisant à un coût de la tonne de fer pur voisin de celui du rond à béton). Ces études sont également reprises par la Kawasaki Steel Corp [158], la Foster Wheeler $[159,160]$ et tout récemment par SKF en Suède.

- La production de silicium ultrapur pour photopiles solaires est également testée en plasma par Westinghouse à l'échelle de $1 \mathrm{MW}$ en utilisant le sodium comme réducteur $[161,162]$.

- Les plasmas peuvent également servir à valoriser certains minerais par évaporation, notamment :

- décomposition de silicates : manganèse [163], beryl [164, 165],

- récupération d'étain dans les laitiers par évaporation $[166,167]$,

- récupération de plomb dans les bains à base de silicate de plomb [168],

- récupération de fer à partir de bauxite [169],

- récupération de manganèse dans des bains à base de silicate de manganèse [170].

- On peut également envisager la sphéroïdisation des argiles en plasma [171], la fusion de divers réfractaires dans des fours rotatifs à axe horizontal [172-175] ou vertical [176] où le traitement des phosphates 
apatitiques [177] pour leur conférer des propriétés de dissolution contrôlées dans le temps.

\subsubsection{Produits à moyenne ou forte valeur ajoutée. -} a) Les projections par plasma. - Les projections plasma font actuellement l'objet de recherches intensives pour la projection de multi-couches ayant une bonne résistance aux chocs thermiques (couches successives permettant de passer du coefficient de dilatation de la céramique à celui du métal par une série de valeurs intermédiaires). Ceci permettrait par exemple l'utilisation de céramiques à haute température et bas coefficient de frottement comme revêtement interne des moteurs [111].

Par ailleurs les essais développés à l'Institut Paton [111] ont montré la potentialité de la projection plasma pour réaliser la matrice de matériaux composés à base de fibres ou de wiskers avec une forte adhésion de la matrice aux fibres sans pour autant modifier les propriétés de la fibre du fait de la brièveté de l'interaction fibre-particule lors de la projection $\left(10^{-2}-10^{-4} \mathrm{~s}\right)$.

b) Les traitements de surface. - Il faut d'abord rappeler la technique de bombardement ionique qui permet par exemple de nitrurer des aciers [178]. En plasma basse pression, la nitruration du titane lui confère des propriétés de frottement comparable aux aciers et permet d'envisager son utilisation dans des appareillages mécaniques où l'on recherche un allègement de la masse et une tenue en température.

Naturellement, toutes les techniques plasma en électronique déjà citées sont en plein développement, tant pour le dépôt des couches que pour leur gravure. Outre les travaux sur l'étude fondamentale des phénomènes mis en jeu tels que la synergie de la chimisorption et des espèces chargées [179-181], sur la modélisation [182], la technologie des réacteurs s'affine [183, 184].

Il faut noter que les processus de polymérisation utilisés dans les techniques plasma pour l'électronique dépendent du contrôle de la densité et de l'énergie des électrons dans la décharge [180] et des phénomènes de chimisorption [185], divers composés étant actuellement testés (organosilicones [186], éthane [187], méthyl-méthacrylate [188], éthylène [189]).

Il faut aussi indiquer que les techniques plasma permettent par réaction en phase gazeuse suivie d'une trempe contrôlée sur une paroi froide, de produire $\mathrm{du}$ silicium amorphe intéressant pour les problèmes de captation de l'énergie solaire par photopile. Par ailleurs, les premières études semblent indiquer qu'il serait possible en chimie des plasmas d'obtenir directement des couches minces de silicium [190, 191].

De même il est possible d'améliorer les fibres naturelles ou artificielles à usage de l'industrie textile afin de leur conférer des propriétés de mouillabilité, de résistance mécanique et de coloration compatibles avec leur emploi habituel [192].
Les techniques de dépôts par exemple par ion plating sont également très prometteuses mais les publications que l'on trouve se rapportent encore surtout à des résultats obtenus en laboratoire. Nous allons rappeler les principales :

- traitements anti-usure : protection des outils de coupe par des couches de 2 à $20 \mu \mathrm{m}$ d'épaisseur de carbure, de nitrure ou de carbo-nitrure de titane [193197]. Schintlmeister et Pacher [194] montrent que la durée de vie d'un outil de coupe recouvert de carbure de titane est multipliée par 2. Elle peut être multipliée par 3 ou par 4 s'il est recouvert d'un carbonitrure de titane. Le coût du traitement entraîne une dépense supplémentaire de $20 \%$, on voit donc tout l'intérêt d'un tel traitement.

- Traitements anti-corrosion : dépôts d'alumine [198, 199]; Swaroop et ses collaborateurs [198] afin de protéger des conduites dans les installations de production de gaz de chargin soumises à l'action de gaz corrosifs à haute température et pression élevée, ont réalisé des dépôts d'alumine sur de l'acier. Les épaisseurs des dépôts sont soumises entre 10 et $100 \mu \mathrm{m}$. La vitesse de dépôt est de l'ordre de $900 \mu \mathrm{m} \cdot \mathrm{h}^{-1}$.

- Dépôts métalliques auto-lubrifiants : dépôts de titane, de plomb, d'or ou de nickel [200].

- Dépôts protecteurs et décoratifs : dépôts de nitrure de titane sur les boîtiers de montre [201].

Ce n'est que depuis quelques années que les applications de l'ion plating à l'électronique se développent. Nous pouvons citer :

- Dépôts de métaux purs : dépôt d'or [202]. Cette application devrait être particulièrement intéressante dans le cas de dépôts sur isolants, en particulier dépôts de cuivre ou d'or sans sous-couche d'accrochage [203].

- Dépôts de couches ferromagnétiques de cobalt ou de nickel [204, 205].

- Dépôts réactifs de couches photoconductrices d'oxyde de plomb [206].

- Dépôts réactifs de couches de nitrure et de nitrure de tantale [207].

- Dépôts réactifs de couches transparentes et conductrices d'oxyde d'indium et d'étain (I.T.O. [207]).

- Dépôts réactifs de couches protectrices de nitrure de silicium [208].

- Dépôts de rhénium, de ruthérnium sur les contacteurs de relais $[209,210]$. L'excellente adhérence des dépôts et leur bonne répartition font de l'ion plating un outil de choix pour ce type de revêtements.

c) Synthèses sélectives. - Enfin, un certain nombre de réactions chimiques de synthèse restent encore très difficiles car les espèces susceptibles de réagir ont, soit une concentration trop faible, soit une durée de vie trop brève. Il convient donc, par le biais des transferts d'excitation sélectifs, soit de surpeupler ces niveaux, soit d'isoler des espèces de longue durée 
de vie. On peut par exemple citer la synthèse de $\mathrm{N}_{2} \mathrm{O}$ à partir de l'oxygène $\mathrm{O}\left({ }^{1} \mathrm{D}\right)$ trempé par de l'azote froid (dans l'état fondamental) et la synthèse des médicaments et parfums par l'oxygène singulet $\left(\mathrm{O}_{2}{ }^{1} \Delta_{\mathrm{g}}\right)$ qui permet une stéréochimie très spécifique développée par exemple par l'Air Liquide [211, 212]. Dans cette optique, il convient d'étudier tout particulièrement la production des états métastables et excités atomiques et moléculaires de l'oxygène et de l'hydrogène, ainsi que les réactions spécifiques qu'ils permettent d'obtenir. Il semble d'ailleurs qu'il y ait actuellement des perspectives intéressantes de synthèse de l'hydrazine en plasma par formation du radical $\mathrm{NH}_{2}$ [213-216].

d) Divers. - Mentionnons simplement : l'utilisation des générateurs à arc transféré et fonctionnant sous des pressions jusqu'à 100 bars pour le découpage et le soudage sous la mer [217].

Pour conclure, on peut signaler l'intérêt de la purification du'silicium métallurgique pour obtenir $\mathrm{du}$ silicium solaire par fusion de zone en plasma HF [218].
6. Conclusion. - Nous avons présenté sommairement les diverses techniques plasma tant thermiques qu'hors d'équilibre utilisées en chimie des plasmas où pour les traitements de surface. Un nombre encore réduit d'applications est maintenant passé au stade industriel, essentiellement d'ailleurs pour des applications spécifiques où la technique plasma est sans concurrence. Cependant on voit dès maintenant certaines techniques plasma se substituer aux techniques classiques soit parce qu'elles sont plus simples et moins onéreuses, soit parce qu'elles sont moins polluantes. Cependant si l'utilisation industrielle des plasmas est en plein développement (voir les applications prospectives que nous avons recensées), elle est encore largement inexplorée (citons par exemple la préparation de poudres ultrafines pour certains frittages, etc...). On peut donc espérer la voir se développer dans la mesure où les phénomènes seront mieux compris, ce qui conduira à l'abaissement des coûts énergétiques et à l'augmentation des rendements de conversion. Il y a là encore un vaste programme de recherche pour des équipes pluridisciplinaires.

\section{Bibliographie}

[1] Edstrom, J. S., Petrochem. Ind. 2 (1904) 399

[2] Baronnet, J. M., Contribution à l'étude spectroscopique des plasmas d'azote produits par un générateur à arc soufflé; application de la chimie des plasmas : synthèse des oxydes d'azote ", Thèse de Doctorat d'Etat, Université de Limoges, 1978.

[3] Cappezuto, P. et al., J. Phys. Chem. 60 (1976) 882.

[4] Catherinot, A., Sy, A., Z. Naturforch. 30a (1975) 1143.

[5] Syduk, V. L., Eremin, E. N., Russ. J. Phys. Chem. 49(4) (1975) 917.

[6] Venugopalan, M., Plasma Chemistry. An introduction. In : Reactions under plasma conditions. Chap. XI, Vol. II, ed. Venugopalan (Wiley Interscience N. Y.) 1971.

[7] GAGE, R. M., Arc torches and process, U. S. Patent 2 (1957) 806, 124.

[8] Pfender, E., Electric Arcs and Arc Gas Heaters, in Gaseous electronic, ed. M. N. Hirsh, HJ Oskam (Acad. Press) 1978.

[9] Coudert, J. F., Contribution à l'étude de la synthèse des oxydes d'azote par chalumeau à plasma, Thèse de $3^{\mathrm{e}}$ cycle, Université de Limoges, 1978.

[10] Kimblin, S. U., « Erosion des électrodes et processus d'ionisation entre les électrodes de l'arc dans le vide et à la pression atmosphérique » p. 226 in Investigations expérimentales des plasmatrons. M. F. Joukov (Ed. : Nauka Novossibirsk) 1977.

[11] Leontiev, A. J., Voltshkov, E. P., Caractéristiques électriques et thermiques d'un plasmatron de haute enthalpie " in "Investigations expérimentales des plasmatrons» M. F. Joukov (Ed. Nauka Novossibirsk) 1977.

[12] Joukov, M. F., Kourotchkine, Poustogarov, A. V., Hydrodynamique appliquée des plasmas thermiques, Rédacteur : Koutadieladze S. S. (Ed. Nauka Novossibirsk) 1975.

[13] Joukov, M. F., Kourotchkine, Poustogarov, A. V., «Etude des plasmatrons utilisant des gaz plasmagènes soufflés à travers un étage rapporté entre les électrodes " p. 82 in Investigation expérimentale des plasmatrons, M. F. Joukov (Ed : Nauka Novossibirsk) 1977.

[14] Schnell, C. R., Hamblyn, S. M. L., Hengarther, K. and WissLER, M. (Lonza Ltd), The industrial application at the Symposium Commercial Potential for Arc and Plasma Processes, Atlantic City, N. J., 8-11 septembre (1974).

[15] Fey, M. G., Kemeny, G. A., Method of direct ore reduction using a short gap arc heater, U. S. patent 3, 765, 870, oct. 16, 1973.

[16] Bonet, C. et al., J. Phys. D. 9 (1976) L-141.

[17] Shakov, M. F., Smolyakov, V. Ya, Urgukov, B. A., Electric arc heaters of gases (ed. Nauka Moscow) 1973.

[18] Fiszdon, J., LesinSKi, J., International Round Table on Study and Application of Transports Phenomena in Thermal Plasmas, Odeillo, 12-16 sept. 1975.

[19] Capitelli, M., Gorse, C., Fauchais, P., J. Chim. Phys. 7-8 (1976) 755.

[20] Rykalin, N. N., Plasma engineering in metallurgy and inorganic materials technology, Plasma Chemistry 2, IUPAC (Pergamon Press) 1977, p. 179-194.

[21] Sayce, I. G., Plasma Processes in extractive metallurgy, in the proceedings of Symposium on Advances in Extractive Metallurgy and Refining, London, octobre 4-6 1971.

[22] Hamblyn, S. M., Minerals Sci. Eng. 9 (1977) 151.

[23] Gold, D., Revue de la technologie des plasmas thermiques. Nouvelles applications de l'Electricité, Colloque international, Toulouse 7-11 mars 1977, Contribution VI-3.

[24] Fauchais, P., Bourdin, E., Ceramics and plasma, in the proceedings of the III International Symposium on Plasma Chemistry, Limoges 13-19 july 1977, Invited Paper.

[25] BonET, C., Le four à plasma en minéralurgie et en métallurgie extractive. Journée d'Etude de la SEE jeudi 27 avril 1978.

[26] Maecker, H., Z. Physik 136 (1953) 119.

[27] Bhattacharya, D., Gauvin, W. H., J. Appl. Phys. 47 (1976) 4863.

[28] Wilks, P. H. et al., Chem. Eng. Prog. 68 (1972) 82.

[29] Sheer, C., Korman, S., KANG, S. F., Investigation of convective arcs for the simulation of re-entry aerodynamic heating. AFOSR-TR-74-1505, Contrat F-44 620-69-C0104 (1974).

[30] Bayliss, R. K., Bryant, J. W., Sayce, I. G., Plasma dissociation of zircon sands, in the proceedings (S-5-2) of 
the III International Symposium on Plasmas Chemistry, Limoges, France 12-19 july 1977.

[31] KubaneK, G. R., Munz, R. J., Gauvin, W. H., Plasma decomposition of molybdenum disulphide. A progress report, in the proceedings (S-5-4) of the III International Symposium on Plasma Chemistry, Limoges, 13-19 july 1977.

[32] Bonet, C., Vallbona, G., Foex, M., Daguenet, M., DumarGUE, P., Rev. Int. Hautes Temp. Réfract. 11 (1974) 11.

[33] Pickles, C. A., Wang, S. S., Mclean, A., Alcock, C. B., Segworth, R. S., Trans. Iron Steel Inst. Japan 18 (1978) 369.

[34] Whyman, D., J. Sci. Instrum. 44 (1967) 525.

[35] Tylko, J. K., High Temperature Treatment of Materials, Can. Pat. no 957733, Granted to Tetronics Ltd., 1974.

[36] Arc-Coal Process Dev., Final Report may 1966, april 1972, AVCO Corporation System Division, Towell, Massachusetts, USA 91851.

[37] Sayce, I. G., Selton, B., Spec. Ceram., British Ceramics Research Association 5 (1972) 157.

[38] Yerouchalmi, D. et al., High Temp. High Pressures 3 (1971) 271.

[39] Howie, F. H., SAyce, I. G., Rev. Int. Hautes Temp. Réfract. 11 (1974) 169

[40] Chase, J. D., Skriven, J. F., Process for the benefication of titaniferous ores utilising a hot wall continous plasma reactor, U.S. Pat. 3 (1974) 856, 918.

[41] Gold, R. G., Sandall, W. R., Cheplick, P. G., Mac-Rae, D. R., Plasma reduction of iron oxide with hydrogen and natural gas at $100 \mathrm{~kW}$ and $1 \mathrm{MW}$, International Round Table on Study and Application of Transport Phenomena in Thermal Plasmas, IUPAC Odeillo 12-16 sept. 1975 proceedings.

[42] Magnolo, G., Can. Min. Metall. Bull. 57 (1964) 57.

[43] FiEdLER, H. et al., Results of plasma melting of steel, Fifth International Symposium on Electroslag Remelting and Other Special Melting Process Pittsburg, 1974.

[44] Mackinnon, I. M., Reuben, B. G.; J. Electrochem. Soc. 122 (1975) 806.

[45] Roman, W. C., Zabielski, M. F., Spectrometric Gas Composition Measurement of $\mathrm{UF}_{6} \mathrm{RF}$ Plasmas. Presented at 30th Annual Gaseous Electronics Conference, Palo Alto, CA, oct. 1977.

[47] ECKert, H. U., High Temp. Sci. 6 (1974) 99.

[48] Boulos, M. I., $3^{\mathrm{e}}$ Symposium International de Chimie des Plasmas IUPAC, Proceeding S-3-2, Limoges, ed. P. Fauchais, 1977.

[49] Rykalin, N. N., Pure Appl. Chem. 48 (1976) 179.

[50] Hirsh, M., Décharges HF et microondes, Séminaire Chimie des Plasmas, Dourdan 20-24/02/78, ed. A.D.E.R.P. Orsay.

[51] Fehsenfeld, F. C., Evenson, K. M., Broida, H. P., Rev. Sci. Instrum. 36 (1955) 294.

[52] Hubert, J., Moisan, M., Ricard, A., Spectrochim. Acta 33 (1979) 1 .

[53] Muller, K. G., Non equilibrium plasmas and their diagnostics, invited paper of the Third International Symposium on Plasma Chemistry, Limoges july 13-19 1977.

[54] Salge, J. and BraumanN, P., 4th International Symposium on Plasma Chemistry, Proceedings p. 735, Zurich, ed. Veprek S., 1979.

[55] Reinberg, A. R., Plasma Deposition of Inorganic Silicon containing films, invited paper in International Round Table on Surface Treatment and Polymerisation in plasmas, Limoges 11-12/07 1977, ed. P. Fauchais.

[56] Hollahan, J. R., Rosler, R. S., in Thin Films Processes, J. L. Vossen and W. Kern ed. (Academic Press, NY) 1978 , p. 335.

[57] Yasuda, H., in Thin Films Processes, J. L. Vossen and W. Kern ed. (Academic Press, NY) 1978, p. 361.

[58] Melliar-Smith, C. M. and Mogab, C. J., in Thin Films Processes, J. L. Vossen and W. Kern ed. (Academic Press, NY) 1978, p. 497.
[59] Vossen, J. L., J. Electrochem. Soc. 126 (1979) 319.

[60] Mattox, D. M., J. Vac. Sci. Technol. 10 (1973) 47.

[61] Teer, D. G., J. Phys. D 9 (1976) 187.

[62] TeER, D. G., Surfacing Journal, oct. 1977, 11.

[63] Venugopalan, M., Plasma Chemistry. An introduction, chap. XI, Vol. II dans Reactions under Plasma conditions, Ed. par Venugopalan (Wiley Interscience) 1975.

[64] Baronnet, J. M., Coudert, J. F., Rakowitz, J., Bourdin, E., FauchaIs, P., 4e Symposium International de Chimie des Plasmas, Proceeding p. 349, ed. Veprek S. (Zurich) 1979.

[65] Eliasson, B., Kogel Schatz, U., 4e Symposium International de Chimie des Plasmas, Proceedings p. 729, ed. Veprek S. (Zurich) 1979.

[66] Fauchais, P., Rakowitz, J., Physics on Plasma Chemistry, J. Physique Colloq. 40 (1979) C7-289.

[67] Polak, L., Some principles of non equilibrium plasma chemical reaction kinetics, Chap. 13, p. 141, T. 2, Plasma Chemistry, ed. Venugopalan (Wiley-Interscience, N.Y.) 1971.

[68] Winters, H. F., Elementary processes at solid surfaces immersed in low pressure plasmas, in 4th International Symposium on Plasma Chemistry, 1979, Univ. of Zurich, ed. S. Veprek, Proceedings p. 28.

[69] Hudis, M., Plasma Treatment of Solid Materials in Techniques and Applications of Plasma Chemistry, ed. J. R. Hollahan et A. T. Bell (Wiley Interscience) 1974.

[70] Zarowin, C. B., A theory of plasma chemical transport in 4th International Symposium on Plasma Chemistry, 1979, Univ. of Zurich, ed. S. Veprek, Proceedings p. 56.

[71] Rapakoulias, D., Etude des processus de fixation de l'azote dans un réacteur chimique à plasma hors d'équilibre. Mise en évidence de processus catalytique, Thèse de Doctorat ès Sciences Physique, Université de Paris VI, 11/06/1979.

[72] Holland, L., Sidall, G., Vacuum 3 (1953) 375.

[73] Bessot, J. J., La pulvérisation cathodique, mise en axuvre et applications, note technique Alcatel, 1970.

[74] Chapin, J., Schaeffer, K., 2e Colloque International de Pulvérisation cathodique, Nice, 1976, pp. 268-270.

[75] Patz, W., Flaming, A., Thin Solid Films 51 (1978) 297.

[76] Machet, J., Guille, J., Colloque Européen Surfaces, Vide, Métallurgie, Strasbourg 9-12 mai 1978, Vide, no spécial, pp. 155-161.

[77] Plas, F., Guille, J., Machet, J., C.I.P. 79, Nice 11-14 sept. 1979, pp. 45 à 52.

[78] Jones, C. K. and Martin, S. W., Met. Prog. 85 (1964) 95.

[79] Hudis, M., J. Appl. Phys. 44 (1973) 1489.

[80] VEPREK, S., A theoretical approach to heterogeneous reactions in non-isothermal low pressure plasma, in Topics in Current Chemistry, vol. 56 (Springer, Berlin) 1975.

[81] WIRZ, E., Ph. D. Thesis, University of Zurich, 1975.

[82] Bradley, A. and Heagney, T. D., Anal. Chem. 42 (1970) 894.

[83] Rossmann, K., J. Polymer. Sci. 19 (1956) 141.

[84] Hudis, M., Plasma Treatment of Solid Materials in Techniques and Applications of Plasma Chemistry, ed. J. R. Hollahan et A. T. Bell (Wiley Interscience) 1974.

[85] ReInberg, A. R., Plasma deposition of inorganic silicon containing films, invited paper, International Round Table on Plasma Polymerisation and Surface Treatment, Limoges 11-12 july 1977, ed. Prof. Fauchais.

[86] Taggert, Mc F., Plasma Chemistry in Electrical Discharges (Elsevier Publishing Co) 1967.

[87] Poulsen, R. G., J. Vac. Sci. Technol. 14 (1977) 266.

[88] ABE, H., Present and future aspect of dry process in semiconductor device manufacture, invited paper, International Round Table on Plasma Polymerisation and Surface Treatment, Limoges 11-12 july 1977, ed. Prof. Fauchais.

[89] Tномаs, R. S., Use of chemically reactive gaseous plasmas in preprations of specimens for microscopy, in Techniques 
and applications of plasma chemistry, ed. Hollahan J. R. and Bell A. T. (Wiley Interscience) 1974.

[90] Maisonneuve, J. M., Thèse de Docteur-Ingénieur, Toulouse (1975).

[91] Fey, G., Electric arc heater for the process industries. National Industrial Electric Heating Conference Cincinnati, Ohio USA, feb. 9-12-1976.

[92] Dumon, A., Inf. Chim. 140 (1970) 69.

[93] SpItz, P. H., Inf. Chim. 164 (1977) 101.

[94] Chim. Actual. 1523 (1974) 19.

[95] WiLKs, P. H. and THORPE, M. L., The heating of solids in high temperature plasma. Paper presented at American Chemical Society, Symposium on high temperature, Chicago, 1970, $13 \mathrm{p}$.

[96] Evans, A. M., Williamson, J. P. H., J. Mater. Sci. 12 (1977) 779.

[97] Evans, A. M., Williamson, J. P. H., J. Mater. Sci. 14 (1979) 680.

[98] Fry, M. et al., Spheroidization of magnetite using an A.C. arc heater, International Round Table on Study and application of transport phenomena in thermal plasmas. Proceedings, Odeillo sept. 1975.

[99] Schnell, C. R. et al., The industrial application of plasma technology for the production of fumed silica, Symposium on Commercial Potential for Arcs and Plasma Processes, Atlantic City, N. J., 8-11 septembre 1974.

[100] LANDT, U. et al., Production of smouth and spheroidal ferrosilicon particles, S. A. Pat. 720850, appl., 1971.

[101] Rykalin, N. N., Pure Appl. Chem. 48 (1976) 133.

[102] Borodatchov, A. S. et al., Fours d'aciérie à plasma à creuset céramique. Etude et résultats d'exploitation, Congrès d'électrotechnique mondial, 21-25 juin 1977, Moscou, Section 4A, rapport 26.

[103] Asada, C. et al., Plasma Induction Heating. Proceedings Third International Symposium on Electroslag Melting Processes, Pittsburg USA, 1971.

[104] Paton, B. et al., Plasma arc remelting in copper watercooled crystalliser as a new method of improving metal and alloy properties. Proceedings Third International Symposium on Electroslag Melting Processes, Pittsburgh USA, 1971.

[105] Kinoshito, T., Shinku, 17 (1975) 61 (en japonais).

[106] SchoumakeR, H. R. P., Fours à chauffage plasma dans "Conference proceedings of International Round Table on Study and Application of Transport Phenomena in Thermal Plasmas ", laboratoire des Ultra-Réfractaires du C.N.R.S., Odeillo sept. 1975.

[107] Rossener, H. O., Stahl Eisen 95 (1975) 61.

[108] Arkless, K., Ceaver, D., Brit. Patent, 1226 082, 1966.

[109] Sayce, I. G., Pure Appl. Chem. 48 (1976) 215.

[110] Korman, S., Sheer, C., Electrochem. Soc. Symp. Philadelphia, 1966.

[111] Rykalin, N. N., Kudinov, V. V., Pure Appl. Chem. 48 (1976) 229.

[112] Besson, J. L., Boch, P., Plasma Spraying of Ceramics, International Round Table Discussion on "Special Ceramics for Electronics and Electrical Engineering », Warsaw $8-11$ oct. 1978

[113] Besson, J. L., Vardelle, M., Boch, P., Ind. Céram., 1979.

[114] Ingham, H. S., Shepard, A. D., Meteo Flame Spray Handbook, 1965.

[115] Kumar, R., Ladas, C., Hudson, G., Characterization of Plasma Etching for Semiconductor Applications, Solid State Technol., october 1976, p. 54-59.

[116] Bessot, J. J., La pulvérisation cathodique, mise en auvre et applications, note technique Alcatel, 1970.

[117] Groset, M., Perez de la Sota, R., Velasco, G., LassaBATERE, L., $I^{\text {er }}$ Colloque International de pulvérisation cathodique, Montpellier, oct. 1973, p. 65-70.

[118] Francombe, M. H., $1^{\mathrm{er}}$ Colloque de pulvérisation cathodique, Montpellier, oct. 1973, pp. 81-91.
[119] Daviose, P. D., Maissel, L. I., J. Appl. Phys. 37 (1966) 574

[120] Auberger, G., DAvid, R., $1^{\mathrm{er}}$ Colloque International de Pulvérisation cathodique, Montpellier, 1973, pp. 295-299.

[121] Pichorr, R., 2 $2^{\mathrm{e}}$ Colloque International de Pulvérisation cathodique, Nice, 1976, pp. 133-136.

[122] BuIsINE, A., Traitement industriel du polyéthylène par effet couronne, A.D.E.R.P. Chimie des Plasmas, Dourdan fév. 1978

[123] Амоuroux, J. et al., Propriétés adhésives des films de polyéthylène soumis à l'action d'une décharge couronne, A.D.E.R.P. Chimie des Plasmas, Dourdan fév. 1978

[124] Gladish, H., Hydrocarbon Process. Pet. refines 41 (1962) 159.

[125] Gladish, H., Chimie Ingénieur Tecknik 41 (1969) 204.

[126] Dupont, Arc Acetylene Process par R. A. Schulze, Chem. Ind. 9 (1968) 1539.

[127] ARC-COAL, Process Development, Final report may 1966 april 1972, AVCO CORP. Syst. Div. Lowell Massachusetts USA 01851 .

[128] BaBCock, J. A., Design engineering of an arc acetylene plant, Chem. Eng. Process. 71 (1975) 90.

[129] Rapakoulias, D., Amouroux, J., Etude de la catalyse dans un réacteur chimique à plasma hors d'équilibre I. Système $\mathrm{N}_{2}-\mathrm{CH}_{4}$. $4^{\mathrm{e}}$ Symposium International de Chimie des Plasmas, Zurich (1979), p. 372.

[130] Drost, H., Behlke, H., Spangenberg, J., Sensitized Conversion of Methane to Acetylene and Ethylene by Additions of Hg Vapour in a Thermal Plasma. 4e Symposium International de Chimie des Plasmas, Zurich (1979), p. 402.

[131] Eremin, E. N., Mal'tsev, A. N. et Rusakova, L. A., Russ. J. Phys. Chem. (1974) 1256 et (1974) 1129.

[132] Mal'tsev, A. N., Eremin, E. N. et Belova, V. M., Russ. J. Phys. Chem. (1971) 1042

[133] Polak, L. S. (red), Kinetika i thermodinamika Khimiceskikh protsessov V nizkotemperatournoy Plazmz. (Ed. Nauka, Moscou) 1965.

[134] Pollo, I., 3e Symposium International de Chimie des Plasmas, IUPAC Limoges G-1-9-1977.

[135] Coudert, J. F., Baronnet, J. M., Rakowitz, J. et Fauchais,

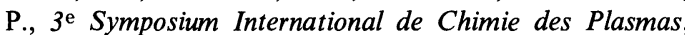
IUPAC Limoges G-1-7-1977.

[136] Amouroux, J., Cavadias, S., Rapakoulias, D., 3e Symposium International de Chimie des Plasmas, Limoges G-1-5-1977.

[137] Cavadias, S., Rapakoulias, D., Amouroux, J., Etude de la catalyse dans un réacteur chimique à plasma hors d'équilibre II. Système $\mathrm{N}_{2}-\mathrm{O}_{2}$. $4^{\mathrm{e}}$ Symposium International de Chimie des Plasmas, Zurich, p. 378, 1979.

[138] Locoueneux, M., Etile, F., Ben-Aïm, R. I., Synthesis of Nitrogen Oxides in a Microwave Discharge. $4^{\mathrm{e}}$ Symposium International de Chimie des Plasmas, Zurich, 1979, p. 366.

[139] Ricard, A., Etile, F., Locoueneux, M., Spectroscopic Study of $\mathrm{N}_{2}-\mathrm{O}_{2}$ High Pressure UHF Plasma. 4e Symposium International de Chimie des Plasmas, Zurich, 1979, p. 652.

[140] Baronnet, J. M., Coudert, J. F., Rakowitz, J., Bourdin, E., Fauchais, P., Nitrogen Oxides Synthesis in a DC plasma Jet. $4^{\mathrm{e}}$ Symposium International de Chimie des Plasmas, Zurich, 1979, p. 349.

[141] Pollo, I., Koffmann, K., Fedenszuk, L., Development of Nitric Oxide Synthesis in a DC plasma. Chemical Reactor. $4^{\mathrm{e}}$ Symposium International de Chimie des Plasmas, Zurich, 1979, p. 361

[142] Jones, K., The Chemistry of Nitrogen (Pergamon Press) 1975.

[143] Lachmann, J. et al., Z. Phys. Chem. (Leipzig) 255 (1974) 1048.

[144] Freeman, M. P., Evans, N. E. V., Winkler, C. A., Can. J. Chem. 34 (1956) 1271.

[145] Munz, R. J., Gauvin, W. H., AI Ch EJ 12 (1975) 1132.

[146] Boulos, M. I., Gauvin, W. H., Powder Processing in a Plasma Jet : proposed model., Can. J. Chem. Eng. 52 (1974) 355 . 
[147] Kubanek, G. A., Munz, R. J., Gauvin, W. H., Plasma decomposition of Molybdenum disulphide, A progress report, III Symp. Int. de Chimie des Plasmas, IUPAC Limoges, 13-19 juillet 1977 .

[148] Kubanek, G. R., Gauvin, W. H., Irons, G. A., Choi, H. K., The Industrial Application of Plasmas to Metallurgical Processes. $4^{\mathrm{e}}$ Symposium International de Chimie des Plasmas, Zurich, 1979, p. 247.

[149] MAC RAE, D. R. et al., Ferrovanadium production by plasma carbothermic reduction of vanadium pentoxide, XXXIV Conf. Electric. Furnace, St. Louis, USA, déc. 1976.

[150] MAC RAE, D. R. et al., "Ferrovanadium production by plasma carbothermic reduction of vanadium oxide». T. III, Third Int. Symp. on Plasma Chemistry, IUPAC Limoges, 13-19 juillet 1977.

[151] Fey, M. G., Harvey, F. J., Plasma heating devices in the electric energy economy, Met. Eng. $Q$., may 1976, 27-30.

[152] Wolf, G. B., Westinghouse Electric. Private Communication, feb. 1976.

[153] Pickles, C. A., Wang, S. S., Mc Lean, A., Alcock, C. B., Segsroorth, P. S., IEEE Trans. 18 (1978) 369.

[154] Kassabi, F., Pateyron, B., Aubreton, J., Fauchais, P., Amouroux, J., Morvan, D., Technical and economical studies for metal production by plasma steel making application, IVth International Symposium on Plasma Chemistry, Zurich aug. 79, p. 236.

[155] Gold, R. G., Sandall, W. R., Cheplick, P. G., Mac Rae, D. R., Plasma Reduction of Iron Oxide with hydrogen and natural gas at $100 \mathrm{~kW}$ and one megawatt, International Round Table on « Study and application of transport phenomena in thermal plasmas », IUPAC, Odeillo 12-16 septembre 1975, proceedings.

[156] Clump, W. C., Kwasnoski, D., Mac Rae, D. R., Thompson, C. D., A study of high temperature methane reforming, Interamerican Congress of Chemical Engineering Caracas, Venezuela, 13-16 (1975) 33.

[157] Aubreton, J., Fauchais, P., Amouroux, J., Les fours à plasma en métallurgie extractive, Journées d'Etudes, l'Industrie des Métaux, Comité Français d'Electrothermie, Versailles, 5-6 avril 1978.

[158] Mathiesen, M., The 1975 Vallak-Aikoh Scholarship, Stockholm, Sweden, Royal Inst. for Tech., Diun. Ferr. Metall. Report $n^{\circ}$ 521/76, 1976.

[159] TyLko, J. K., The in-flight manufacture of irons and steels in an expanded precessive plasma reactor. III Symposium International de Chimie des Plasmas, IUPAC, Limoges, 13-19 juillet 1977, t. III.

[160] Foster WheELER LTD/TeTronics LTD, «The production of steel from coal and ion core using the Tetronics expanded precessive plasma ". Technical report, dec. 1976.

[161] Fey, M. G., Meyer, T. N., Reed, W. H., An Electric Arc Heater Process to Produce Solar Grade Silicon. 4e Symposium International de Chimie des Plasmas, Zurich, 1979, p. 708.

[162] Heberlein, J. V. R., Lowry, T. N., Meyer, T. N., CiliVERTI, D. F., The production of Tetrachlorosilane by Sodium at High Temperatures in a Laboratory Scale Experiment. 4e Symposium International de Chimie des Plasmas, Zurich, 1979, p. 716.

[163] H HrRis. V. et al.. Arc decomposition of rhodonite, J. Electrochem. Soc. 106 (1959) 874-6.

[164] Bayliss, R. K. and DeRry, R., High-temperature studies in the extraction of beryllium, J. Appl. Chem. 16 (1966) $114-21$.

[165] Everest, D. A., Napier, E. and Wells, R. A., Processes for the extraction of beryllium from flotation concentrates of beryl. In High temperature refactory metals, Krinvsky, W. A. ed. (New York : Gordon and Breach) 1978, 113-28 (Metall. Soc. Conf. Proc. 34 pt 1).

[166] Shelley, T. R. and Charles, J. A., Arc electrolysis of complex tin-containing oxide melts. Trans. Inst. Metall. (Sect. C : Mineral Process. extr. Metall.) 79 (1970) C259-68.
[167] BaretT, M. F. et al., Trans. Inst. Min. Metall. 84 (1975) C-231. [168] Shelley, T. R. and Charles, J. A., Arc electrolysis of lead silicate melts. Trans. Inst. Min. Metall. (Sect. C : Mineral Process. Extr. Metall.) 78 (1969) C-177-80.

[169] Recasens, J., Bortaud, P. and Bonnier, E., Fusion et électrolyse d'oxydes métalliques réfractaires, Rev. Hautes Temp. Refract. 4 (1967) 281-8.

[170] Demoustiez, A., Hanon, J. and Winand, R., Production of manganese by electrolysis of fused oxide mixtures. Industrie Chim. Belge 32 144-55.

[171] Bonet, C., Valbona, G., Foex, M., Bull. Soc. Fr. Céram. 94 (1972) 15256.

[172] Grosse, A. V. et al., Mater. Res. Stand. 5 (1963) 173.

[173] Foex, M., Delmas, R., C. R. Hebd. Séan. Acad. Sci. Paris C1 (1967) 9.

[174] Hamblyn, S. M. L. et al., The industrial application of plasma technology for the production of fumed silica, Symposium of the American Chemical Society on the Commercial Application of Plasma, Atlantic City, 1974.

[175] Yerouchalmi, D. et al., High Temp. High Pressures 3 (1971) 271.

[176] Sayce, I. G., Selton, B., Spec. Ceram., British Ceramics Research Association 5 (1972) 157.

[177] Burov, I. S., Bysyuk, V. V., Zabrodin, V. K., Mosse, A. L., Rev. Hautes Temp. Réfract. 15 (1978) 201.

[178] Gantors, M., Industrie des Métaux, Etudes et Recherches $\mathrm{n}^{\circ} 1,1976$.

[179] Winters, H. F., Elementary Processes at Solid Surfaces Immersed in Low Pressure Plasmas, $4^{\mathrm{e}}$ Symposium International de Chimie des Plasmas, Zurich, 1979, p. 28.

[180] Kay, E., Plasma Polymerization and Etching Mechanisms in Fluorocarbon Systems, 4e Symposium International de Chimie des Plasmas, Zurich, 1979, p. 30.

[181] Wagner, J. J., Brandt, W. W., Plasma Etching of Si by $\mathrm{SF}_{6}, \mathrm{CF}_{4}$ and $\mathrm{SF}_{6} / \mathrm{H}_{2}$ and $\mathrm{CF}_{4} / \mathrm{H}_{2}$ Mixtures. $4^{\mathrm{e}} \mathrm{Sym}$ posium International de Chimie des Plasmas, Zurich, 1979, p. 120.

[182] Zarowin, C. B., A Theory of Plasma Chemical Transport. $4^{\mathrm{e}}$ Symposium International de Chimie des Plasmas, Zurich, 1979, p. 56.

[183] Curtis, B. J., Brunner, H. R., An Optical Detector for Monitoring Plasma Etching. 4e Symposium International de Chimie des Plasmas, Zurich, 1979, p. 139.

[184] Vossen, J. L., Plasma Deposition and Etching Reactors for Semiconductor Applications. $4^{\mathrm{e}}$ Symposium International de Chimie des Plasmas, Zurich, 1979, p. 344.

[185] Kaganowicz, G., Datta, P., Robinson, J. W., The properties of Fluorocarbon Films Prepared by plasma Polymerization of 1,3-Perfluorodimenthylcyclohexane. $4^{\mathrm{e}}$ Symposium International de Chimie des Plasmas, Zurich, 1979, p. 152.

[186] Wrobel, A. W., Wertheimer, M. R., Schieber, H. P., Polymerization of Organosilicones in Microwave Discharges. $4^{\mathrm{e}}$ Symposium International de Chimie des Plasmas, Zurich, 1979, p. 186.

[187] Morita, S., Kawamura, H., Ishibashi, S., Nawata, M., Plasma Polymerization of Hydrocarbon in Afterglow of Argon. 4 $\mathrm{e}^{\mathrm{S}}$ Symposium International de Chimie des Plasmas, Zurich, 1979, p. 748.

[188] Morita, S., Tamano, J., Hattori, S., Ieda, M., Plasma Polymerized Methyl-Methacrylate as an Electron Beam Resist. 4e Symposium International de Chimie des Plasmas, Zurich, 1979, p. 754.

[189] Donohoe, K. G., Wydeven, Polymerization of Ethylene in an Atmospheric Pressure Discharge. 4e Symposium International de Chimie des Plasmas, Zurich, 1979, p. 765.

[190] Turban, G., Catherine, Y., Grolleau, B., Study of the Plasma Deposition Processes of a-Si : H Films. 4e Symposium International de Chimie des Plasmas, Zurich, 1979, p. 164.

[191] Drevillon, B., Huc, J., Lloret, A., Perrin, J., De Rosny, G., SchmitT, J. P. M., A Multipole DC Discharge for 
Thin Film Deposition from Silane Plasma. 4e Symposium International de Chimie des Plasmas, Zurich, 1979, p. 169.

[192] Pavlath, A. E., Landwehr, R. C., Treatment of Fibrous Materials in Glow Discharge. $4^{\mathrm{e}}$ Symposium International de Chimie des Plasmas, Zurich, 1979, p. 486.

[193] Stowell, W. R., Thin Solid Films 22 (1974) 111.

[194] Schintlmeister, W., Pacher, O., J. Vac. Sci. Technol. 12 (1975) 743.

[195] KomiYa, S., Tsuruoka, K., J. Vac. Sci. Technol. 13 (1976) 520.

[196] MukherjeE, J. L., Wu, L. C., Greeene, J. E., CoOK, H. E., J. Vac. Sci. Technol. 12 (1975) 850.

[197] Zega, B., Kornmann, M., Amiguet, J., Thin Solid Films 45 (1977) 577.

[198] Swaroop, B., Meyer, D. E., White, G. W., J. Vac. Sci. Technol. 13 (1976) 680.

[199] Meyer, D. E., White, D. G., Swaroop, B., Thin Solid Films 40 (1977) 319.

[200] Spalvins, T., ALSE Lubr. Eng., february 1971, pp. 40-46.

[201] Zega, M. B., Kornmann, M. M., 52e Congrès de la Société Suisse de Chronométrie, Fribourg, 7-8 oct. 1977, pp. 389391.

[202] Murayana, Y., Japan, J. Appl. Phys., Suppl. nº 2, 1 (1974), pp. 459-462.

[203] HaRker, H. R., Hill, R. J., J. Vac. Sci. Technol. 9 (1972) 1395.

[204] Masatoshi TakaO, AKIRA TASaKi, IEEE, Trans. Magn. 12 (1976) 782.
[205] Hayashi, C., 2 $2^{\mathrm{e}}$ Colloque International de Pulvérisation Cathodique, Nice, mai 1976, pp. 75-89.

[206] Clarke, J. R., Weiss, A. K., Donovan, J. L., J. Vac. Sci. Technol. 14 (1977) 219.

[207] Murayama, Y., J. Vac. Sci. Technol. 12 (1975) 818.

[208] Fukutomi, M., Kitajima, M., OKada, M., Watanabe, R., J. Electrochem., Soc. Solid Science and Technology, sept. 1977, pp. 1420-1424.

[209] Heinz, B., Kienel, G. IPAT 77, Londres 1977, pp. 73-81.

[210] JANNinck, R. F., Heiden, C. R., GutTensohn, A. E., J. Vac. Sci. Technol. 11 (1974) 535.

[211] Dumas, J. L., Garnier, B., J. Chim. Phys. 72 (1975) 1045.

[212] Dumas, J. L., Congrès National de Physique des Plasmas, Colloque "Production de plasma ». Revue Phis. Appl. 12 (1977) 1035.

[213] Carbough, D. C., et al. J. Chem. Phys. 47 (1967) 5211.

[214] Willis, C., Can. J. Chem. 51 (1975) 3605.

[215] Dumas, J. L., Communication privée (1975).

[216] Pannetier, G., Marsigny, L., J. Chim. Phys. 59 (1962) 856.

[217] Waldie, B., Effects of Increasing Pressure on Transferred and Non-Transferred Arcs from a Plasma Cutting Torch. 4 Symposium International de Chimie des Plasmas, Zurich, 1979, p. 311.

[218] Amouroux, J., Morvan, D., Purification du Silicium Métallurgique par une Technique de Fusion de Zone sous Plasma. $4^{\mathrm{e}}$ Symposium International de Chimie des Plasmas, Zurich, 1979, p. 230. 